\title{
New insights into an X-traordinary viral protein
}

\author{
Torsten Schaller ${ }^{1+}$, Hélène Bauby ${ }^{1+}$, Stéphane Hué ${ }^{2}$, Michael H. Malim ${ }^{1}$ and Caroline Goujon ${ }^{1 *}$ \\ Department of Infectious Diseases, King's College London, London, UK \\ ${ }^{2}$ Department of Infection, Division of Infection and Immunity, Centre for Medical Molecular Virology, University College London, London, UK
}

\section{Edited by:}

Nadine Laguette, Centre National de la Recherche Scientifique, France

Reviewed by:

Mikako Fujita, Kumamoto University, Japan

Nathaniel Roy Landau, New York

University School of Medicine, USA

\section{*Correspondence:}

Caroline Goujon, School of

Medicine, Guy's Hospital, Borough

Wing, London SE1 9RT, UK

e-mail: caroline.goujon@kcl.ac.uk

${ }^{\dagger}$ These authors have contributed equally to this work.
Vpx is a protein encoded by members of the HIV-2/SIVsmm and SIVrcm/SIVmnd-2 lineages of primate lentiviruses, and is packaged into viral particles. Vpx plays a critical role during the early steps of the viral life cycle and has been shown to counteract SAMHD1, a restriction factor in myeloid and resting T cells. However, it is becoming evident that Vpx is a multifunctional protein in that SAMHD1 antagonism is likely not its sole role. This review summarizes the current knowledge on this $\mathrm{X}$-traordinary protein.

Keywords: vpx, HIV-2, SIVsmm, SAMHD1, myeloid cells, HIV-1, restriction factor, interferon type I
Vpx was initially identified as an HIV-2 (human immunodeficiency virus type 2)/SIVsmm (simian immunodeficiency virus infecting sooty mangabey monkey) protein of $12-16 \mathrm{kDa}$, which is incorporated into viral particles (Franchini et al., 1988; Henderson et al., 1988; Kappes et al., 1988; Yu et al., 1988). In addition to viruses from the HIV-2/SIVsmm lineage of primate lentiviruses, this gene is also found in viruses from the $\mathrm{SIVrcm}$ (infecting red-capped mangebey)/SIVmnd-2 (infecting mandrill) lineage (Beer et al., 2001; Hu et al., 2003). It is homologous to the vpr gene, found in every lineage of primate lentiviruses (Tristem et al., 1990). Vpx was rapidly shown to be dispensable for viral replication in immortalized lymphocytic cell lines, such as HUT78, CEM, or SupT1 (Yu et al., 1988; Guyader et al., 1989; Hu et al., 1989; Shibata et al., 1990; Gibbs et al., 1994; Park and Sodroski, 1995), and in the monocytic cell lines HL60 and U937 (Guyader et al., 1989; Hu et al., 1989). In contrast, $v p x$ deletion led to a strong replication defect in monocyte-derived macrophages (MDMs) (Yu et al., 1991; Gibbs et al., 1994; Park and Sodroski, 1995; Fletcher et al., 1996; Ueno et al., 2003). In addition, $v p x$ deletion led to SIVmac (infecting rhesus monkey) and HIV-2 replication defects in activated peripheral blood mononuclear cells (PBMCs) or primary $\mathrm{T}$ cells, especially at low viral inputs (Guyader et al., 1989; Kappes et al., 1991; Yu et al., 1991; Akari et al., 1992; Gibbs et al., 1994; Kawamura et al., 1994; Park and Sodroski, 1995; Ueno et al., 2003). Vpx was shown to be important for HIV-2 replication in HSC-F cells, a simian lymphocytic cell line (Ueno et al., 2003). Vpx is packaged into viral particles via an interaction with the p6 domain of Gag (Wu et al., 1994; Accola et al., 1999; Selig et al., 1999) and is associated with mature viral cores (Kewalramani and Emerman, 1996). This suggested that Vpx could participate in the early steps of infection. Comparisons of virus associated proteins suggested that Vpx from SIVmac and HIV-2 are packaged in equimolar amounts to Gag (Henderson et al., 1988), although the exact number of molecules packaged per virion has not been determined.
Vpx localizes to the nucleus in transfected cells (Depienne et al., 2000; Mahalingam et al., 2001; Belshan and Ratner, 2003), and this is conferred by a C-terminal non-canonical nuclear localization signal (NLS) (65-SYTKYRYL-72) (Figure 1) (Belshan and Ratner, 2003; Rajendra Kumar et al., 2003), as well as a potential second N-terminal NLS (Singhal et al., 2006a). Whether Vpx shuttles between the cytoplasm and nucleus due to a nuclear export signal remains controversial (Belshan and Ratner, 2003; Singhal et al., 2006b). Likewise Vpx phosphorylation has been proposed to regulate its nuclear import (Rajendra Kumar et al., 2005) but other studies failed to detect this post-translational modification (Franchini et al., 1988; Belshan et al., 2006). By virtue of its karyophilic properties, $\mathrm{Vpx}$ was proposed to play a critical role in the nuclear import of viral reverse transcription complexes in non-dividing cells, such as MDMs and arrested U937 cells (Pancio et al., 2000; Mahalingam et al., 2001; Rajendra Kumar et al., 2003). Indeed the replication defect of viruses lacking Vpx (or bearing non-karyophilic mutated versions of Vpx) correlated with the absence of 2-LTR circles (a surrogate marker for viral DNA nuclear entry) (Fletcher et al., 1996; Pancio et al., 2000; Ueno et al., 2003; Belshan et al., 2006).

Later studies using lentiviral vectors and single-round infections confirmed a cell-type dependent effect of Vpx and a role in the early events of infection. Vpx is essential for transduction of monocyte-derived dendritic cells (MDDCs) with SIVmac based-lentiviral vectors (Mangeot et al., 2002). Surprisingly, when brought in trans via virus-like particles (VLPs), Vpx increases HIV-1 transduction of MDDCs and MDMs but not activated $\mathrm{T}$ cells (Goujon et al., 2006). This positive effect of Vpx in MDDCs was directly correlated with an increase in viral DNA accumulation, which was observed not only with SIVmac but also with heterologous retroviral vectors, derived from HIV-1, feline immunodeficiency virus (FIV) and murine leukemia virus (MLV) (Goujon et al., 2007). Of note, in the case of MLV, Vpx rescued viral DNA accumulation but not 2-LTR circle formation 


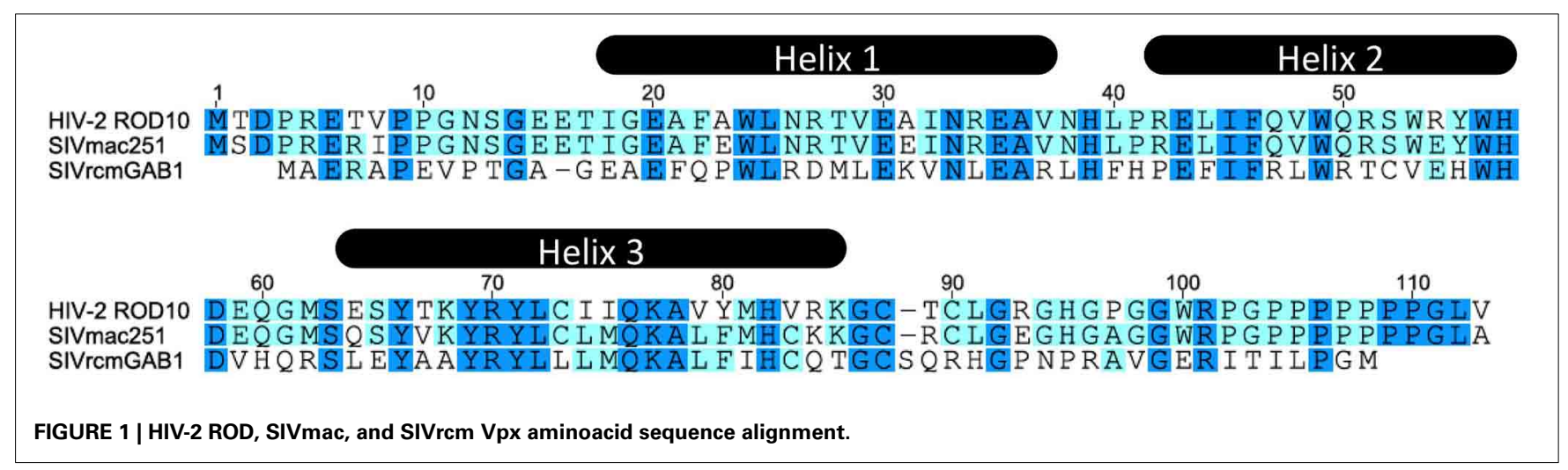

(Goujon et al., 2007; Gramberg et al., 2013), consistent with a nuclear-entry block to MLV infection in non-dividing cells (Roe et al., 1993; Lewis and Emerman, 1994). Vpx was later shown to favor HIV-2/SIVsmm DNA accumulation in MDMs (Fujita et al., 2008; Srivastava et al., 2008; Bergamaschi et al., 2009).

Animal studies showed that Vpx is crucial for SIVsmm PBj and SIVmne (infecting pig-tailed macaque) replication and spread in a pig-tailed macaque model. SIVsmm PBj $v p x$ mutant virus replicated to a considerably lower extent and showed much reduced kinetics as compared to wild-type virus (Hirsch et al., 1998). In addition, the mutant virus was outcompeted when inoculated together with wild-type SIVsmm PBj (Hirsch et al., 1998). In vitro, SIVmne $v p x$ mutants infected pig-tailed macaque PBMCs to comparable levels to that of wild-type virus, but showed significantly reduced infectivity in MDMs (Belshan et al., 2012). Another study reported a less prominent effect of Vpx in rhesus monkeys infected with SIVmac239, with delayed viral kinetics for $v p x$ deficient virus but AIDS development nonetheless (Gibbs et al., 1995).

The fact that Vpx could act in trans on other retroviruses strongly suggested at the time that Vpx modulates the cellular environment to increase cell permissivity to infection, possibly by preventing the action of an inhibitory factor. The first evidence of the existence of such a dominant inhibitory factor in myeloid cells came from the use of heterokaryons generated between permissive (COS) cells and restrictive cells (MDMs) (Sharova et al., 2008). Unlike COS cells, both MDMs and COS-MDMs heterokaryons restricted SIVsmm PBj in the absence of Vpx at the level of viral DNA accumulation. The same authors also observed a dominant restriction phenotype in heterokaryons formed between primary monocytes and HeLa cells (Kaushik et al., 2009).

\section{HIJACKING THE DCAF1/DDB1/CUL4A E3 UBIQUITIN LIGASE}

In parallel with the aforementioned viral and cell biology experiments, proteomic studies yielded some fundamental insights. Initially, Le Rouzic et al. discovered that HIV-1 Vpr recruits the damage-specific DNA binding protein 1 (DDB1)-Cullin 4A (CUL4A) E3 ubiquitin ligase complex through the binding of a known interactor of Vpr, VprBP (Zhao et al., 1994; Le Rouzic et al., 2007). VprBP had previously been identified in a screen as the substrate recruiting module of DDB1-CUL4ARBX1/ROC1 complexes, and renamed DDB1-CUL4A-associated factor 1 (DCAF1) (Angers et al., 2006). Using yeast two-hybrid, Le Rouzic et al. also showed that Vpx from SIVmac, similarly to HIV-1 Vpr, was able to bind VprBP/DCAF1 (Le Rouzic et al., 2007). This interaction was soon confirmed in mammalian cells (Goujon et al., 2008; Sharova et al., 2008; Srivastava et al., 2008; Bergamaschi et al., 2009). RNAi-mediated depletion of DCAF1 or DDB1 profoundly reduced SIVmac as well as HIV-2 infection of macrophages (Sharova et al., 2008; Srivastava et al., 2008; Bergamaschi et al., 2009). This invoked a model by which Vpx was hijacking a DCAF1-DDB1-CUL4A E3 ubiquitin ligase to induce the degradation of a myeloid cell specific restriction factor that prevents viral DNA accumulation (Sharova et al., 2008; Srivastava et al., 2008; Bergamaschi et al., 2009).

\section{SAMHD1 ANTAGONISM}

To isolate the cellular factor(s) limiting HIV-1 infection in myeloid cells, the groups of Monsef Benkirane and Jacek Skowronski exploited similar proteomic approaches, using tandem affinity purification combined with mass spectrometry to identify Vpx binding partners (Hrecka et al., 2011; Laguette et al., 2011). Both labs identified sterile alpha motif (SAM) and HD-domain-containing protein 1 (SAMHD1) as one of the major binding partners of SIVmac Vpx. SAMHD1 silencing phenocopied the effect of Vpx-containing VLPs in that it rescued HIV-1 infection of both differentiated THP-1 monocytic cells and MDDCs (Laguette et al., 2011). Both studies showed that HIV-2/SIVmac Vpx induced SAMHD1 proteasomal degradation (Hrecka et al., 2011; Laguette et al., 2011). Importantly, SAMHD1 antagonism is a function conserved in all clades of Vpx proteins, in a species specific way (Lim et al., 2012). In certain SIVs devoid of a Vpx protein, Vpr can antagonize SAMHD1. For instance, Vpr from SIVdebCM5 (infecting De Brazza's monkeys), SIVagm (African green monkeys), and SIVmus1 (mustached monkeys) can degrade SAMHD1 of their natural host when expressed in human cells, whereas Vpr from HIV-1 and SIVcpz (chimpanzee) cannot (Lim et al., 2012).

SAMHD1 is a 626 amino acid protein that consists of an amino-terminal SAM domain, a central HD domain and a C-terminal uncharacterized domain (Li et al., 2000; Liao et al., 2008). SAMHD1 is a deoxynucleoside-triphosphate (dNTP) phosphohydrolase (Goldstone et al., 2011; Powell et al., 2011; Yan et al., 2013), which reduces the pool of dNTPs available for reverse transcription both in myeloid cells (Lahouassa et al., 2012; 
St Gelais et al., 2012) and in resting T cells (Baldauf et al., 2012; Descours et al., 2012). These observations therefore explain why the provision of exogenous deoxyribonucleosides $(\mathrm{dN})$ to resting $\mathrm{T}$ cells, which have long been known to restrict lentiviral infection at several steps of the life cycle (Pan et al., 2013), increases the accumulation of viral DNA (Korin and Zack, 1999).

Crosslinking experiments show that SAMHD1 forms oligomers in cells and it has been proposed that the enzymatically active form of SAMHD1 is a tetramer and that tetramerization is driven by dGTP binding to the allosteric sites (Ji et al., 2013; Yan et al., 2013). Knock-out mice analysis have recently confirmed that SAMHD1 functions as a dNTPase in vivo as these mice show elevated levels of intracellular dNTPs in DCs isolated from bone marrow (Behrendt et al., 2013; Rehwinkel et al., 2013). Therefore, SAMHD1 reduces cellular dNTP levels and this impacts reverse transcription most significantly in situations where dNTP levels are naturally lower, such as post-mitotic or non-dividing cells.

Mutations in SAMHD1 are associated with the genetic neurodegenerative disorder Aicardi-Goutières syndrome (AGS), which is characterized by the excessive production of type 1 interferon (IFN) in the cerebrospinal fluid and resembles congenital infection (Rice et al., 2009; Chahwan and Chahwan, 2012). Interestingly, CD14+ cells from AGS patients with mutations in SAMHD1 are more susceptible to HIV-1 infection than cells from healthy controls (Berger et al., 2011a). This shows the importance of SAMHD1 for preventing HIV-1 infection in monocytes, well known to be naturally refractory to HIV-1 infection in vitro (Sonza et al., 1996; Neil et al., 2001; Triques and Stevenson, 2004).

In addition to a variety of retroviruses (Gramberg et al., 2013; Sze et al., 2013), SAMHD1 blocks replication of DNA viruses, such as vaccinia virus and herpex simplex virus 1 (Hollenbaugh et al., 2013; Kim et al., 2013). SAMHD1 is also active against retroelements (Zhao et al., 2013), suggesting that increased retrotransposition might be leading to immune sensing of DNA and activation of signaling pathways in AGS patients with SAMHD1 mutations, as proposed for the $3^{\prime}-5^{\prime}$ exonuclease TREX1 (three prime repair exonuclease) (Stetson et al., 2008; Beck-Engeser et al., 2011). Indeed, mutations in TREX1 can cause AGS (Crow et al., 2006) and in Trex1-deficient mice, reverse transcribed DNA from endogenous retroelements accumulated and stimulated intrinsic immune responses (Stetson et al., 2008). Interestingly, Zhao et al. showed that both endogenous and overexpressed SAMHD1 prevented LINE-1 (long interspersed element-1) retrotransposition in 293T cells (Zhao et al., 2013). This effect of SAMHD1 on retrotransposition was not dependent on its catalytic activity and was counteracted by Vpx. Therefore, infection by lentiviruses encoding a SAMHD1 antagonist (either Vpx or Vpr) might lead to increased replication of endogenous retroelements and a possible impact on host genome stability.

SAMHD1 is expressed at similar levels in MDMs, resting CD4+ T cells and in activated CD4+ T cells (Baldauf et al., 2012; Descours et al., 2012), but does not block HIV-1 infection in the latter. The discrepancy of SAMHD1's antiviral function in cycling vs. non-cycling cells led several groups to investigate cell cycle dependent determinants for both the antiviral and dNTPase activities of SAMHD1. SAMHD1 was found to interact with and be phosphorylated by the cell cycle regulator cyclin-dependent kinase 1 (CDK1) in proliferating cells, and phosphorylation at the residue T592 has been shown to prevent lentiviral restriction (Cribier et al., 2013; White et al., 2013). CDK1 is inactive in resting cells, suggesting that the cell cycle progression correlates with SAMHD1's antiviral activity. In line with this, a phosphorylationdefective mutant of SAMHD1 was antiviral both in resting and in dividing U937 cells (Cribier et al., 2013). In addition, the phosphomimetic SAMHD1 mutant T592E was unable to restrict HIV-1 infection (Welbourn et al., 2013; White et al., 2013). However, phosphorylation did not affect the ability of SAMHD1 to hydrolyse dNTPs in an in vitro dNTPase assay (Welbourn et al., 2013) or in differentiated U937 monocytic cells (White et al., 2013). Although phosphorylated and lacking antiviral activity in cycling cells, SAMHD1's role in dNTP metabolism in these cells remains unclear. Cycling cells contain high levels of dNTPs (Diamond et al., 2004), therefore de novo dNTP synthesis likely compensates for any potential effect of SAMHD1. The fact that SAMHD1 mutant T592E lacks antiviral activity while being an active dNTPase suggests the existence of a potential dNTPaseindependent restriction mechanism. Supporting the notion that SAMHD1's influence on infection may be more complex, it has been suggested that SAMHD1 interacts with nucleic acids, specifically ssRNA and ssDNA (Goncalves et al., 2012; Tungler et al., 2013) and that it possesses a nuclease activity (Beloglazova et al., 2013).

SAMHD1 is localized in the nucleus of differentiated cells. However, disruption of its NLS does not affect antiviral activity (Rice et al., 2009; Brandariz-Nunez et al., 2012; Hofmann et al., 2012), yet results in a relative resistance to SIVmac Vpxmediated degradation (Brandariz-Nunez et al., 2012; Hofmann et al., 2012; Wei et al., 2012; Guo et al., 2013). Given that Vpx efficiently interacts with both nuclear and cytoplasmic SAMHD1 (Hofmann et al., 2012), it is difficult to understand why cytoplasmic SAMHD1 is less sensitive to Vpx-induced degradation. It is possible for example that factors of the DCAF1-DDB1-CUL4A E3 ubiquitin ligase machinery are limiting in the cytoplasm, or that differences in SAMHD1's post-translational modifications prevent cytoplasmic SAMHD1 degradation. Alternatively there might be differences in ubiquitination or deubiquitination processes between nuclear and cytoplasmic SAMHD1.

Intriguingly, in contrast to what is observed in MDDCs, wildtype SAMHD1 from myeloid and plasmacytoid DCs (mDCs and pDCs, respectively), was shown to be resistant to Vpx-induced degradation (Bloch et al., 2014). The sensitivity to Vpx-induced degradation of SAMHD1 as well as the Vpx effect on HIV-1 infectivity could be partially restored by blocking IFN signaling using neutralizing antibodies (Bloch et al., 2014). This suggests that IFN-induced factors might prevent SAMHD1 degradation in $\mathrm{mDCs}$ and $\mathrm{pDCs}$ or that SAMHD1 localization might be modified following IFN exposure, rendering it resistant to Vpxmediated degradation. In line with this, Dragin et al. observed a reduced sensitivity of SAMHD1 to Vpx-mediated degradation in IFN-treated THP-1, suggesting that IFN-stimulated genes may participate in this process (Dragin et al., 2013). In addition, IFN treatment did not modify SAMHD1 localization (Dragin et al., 2013). In contrast, we have observed an efficient degradation of SAMHD1 in IFN-treated THP-1 cells (Goujon et al., 2013), but 
the reasons for these differences are currently unknown. Type 1 IFN treatment reduced SAMHD1 phosphorylation levels of residue T592 in MDMs and MDDCs, suggesting the existence of IFN-inducible phosphatase(s) activating SAMHD1 (Cribier et al., 2013). The identification of this/these phosphatase(s) would improve our understanding of the regulation of SAMHD1's antiviral activity.

\section{STRUCTURAL INSIGHTS}

Vpx recruits the DCAF1-DDB1-CUL4A E3 ubiquitin ligase complex to induce SAMHD1's proteasomal degradation via an interaction with its C-terminal domain (Ahn et al., 2012) (Figure 2). The crystal structure of a complex between SIVsmm $\mathrm{Vpx}$, the C-terminal domain of the ubiquitin-ligase adaptor DCAF1 (DCAF1-CtD) and the Vpx-binding, C-terminal domain of SAMHD1 (SAMHD1-CtD, residues 582-626) was revealed recently (Figure 3) (Schwefel et al., 2014). This structure shows that $\mathrm{Vpx}$ is composed of a three-helix bundle stabilized by a zinc finger motif (formed of residues H39, H82, C87 and C89) (Figure 4A) and extensively interacts with DCAF1-CtD (Figure 4B), the latter forming a seven-bladed $\beta$-propeller discshaped molecule. Both proteins offer a shared interface used to bind SAMHD1-CtD. A model of the hijacked DCAF1-DDB1CUL4A complex in association with the RBX1 RING module (RING-box protein 1), generated using previously determined structures, clearly showed that SAMHD1 is positioned in the vicinity of the RBX1 RING module, allowing accessibility to ubiquitination (Schwefel et al., 2014) (Figure 2). The SAMHD1 target sites for $\mathrm{Vpx}$-induced ubiquitination have not been determined yet. In addition to ubiquitination, neddylation is required for SAMHD1 degradation, consistent with the importance of NEDD8 transfer to CUL4A through UBC12 (Hofmann et al., 2013). It would be of interest to understand the structural consequences of Vpx interaction with tetrameric SAMHD1. There is some evidence from surface plasmon resonance experiments that Vpx interaction with SAMHD1 causes the disassembly of enzymatically active SAMHD1 oligomers and interferes with SAMHD1 enzymatic activity prior to its degradation (Delucia et al., 2013). This suggests a model where proteasomal degradation may be the consequence and not the initiating event in Vpx-mediated SAMHD1 antagonism.

Extensive mutagenesis of $\mathrm{Vpx}$ has shed light into the critical residues of Vpx (Table 1 and Figure 4). Notably, N-terminal Vpx amino acids are essential to rescue virus infectivity in myeloid cells and SAMHD1 degradation (Goujon et al., 2008; Gramberg et al., 2010; Ahn et al., 2012; Fregoso et al., 2013). Of major interest, the phenotypes of some well characterized Vpx mutants are explained by the structure (Schwefel et al., 2014). For instance, the Vpx Q76A mutant is unable to bind DCAF1 and degrade SAMHD1 (Srivastava et al., 2008; Bergamaschi et al., 2009; Hrecka et al., 2011). The structure shows that this defect may be due to disrupted hydrogen bonds between Q76 and residues N1135 and W1156 of DCAF1 (Figure 4B) (Schwefel et al., 2014). Similarly, residue K77, critical for DCAF1 binding and Vpx activity (Bergamaschi et al., 2009), is integral to an extensive salt-bridge network that links residues E1091-D1092E1093 of DCAF1 with residues R70, Y69 and Y66 in helix 3 of

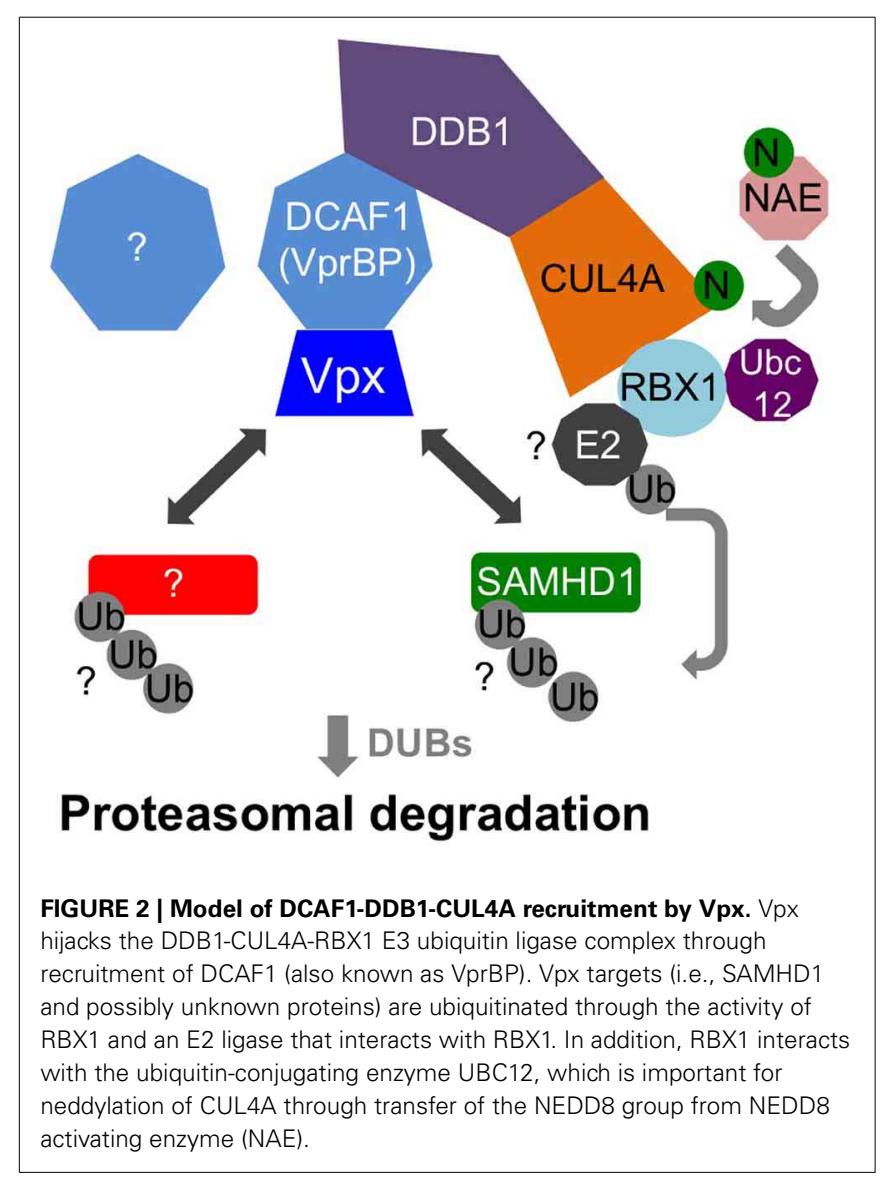

Vpx (Figure 4B). In addition, Vpx W24A mutant was shown to have lost SAMHD1 antagonism while being able to bind to DCAF1 (Wei et al., 2012). This phenotype is explained by a stacking between residues W24 of Vpx and R617 of SAMHD1 (Figure 4C) (Schwefel et al., 2014). In line with this, SAMHD1 R617 mutants completely lost sensitivity to Vpx-mediated degradation while maintaining antiviral activity (Schwefel et al., 2014).

\section{ADDITIONAL ROLES OF Vpx?}

The fact that $v p x$ deletion leads to some replication defects in activated PBMCs or primary T cells (Guyader et al., 1989; Kappes et al., 1991; Yu et al., 1991; Akari et al., 1992; Gibbs et al., 1994; Kawamura et al., 1994; Park and Sodroski, 1995; Ueno et al., 2003) strongly suggests that Vpx might have other functions beyond counteracting SAMHD1, which is only relevant in myeloid and resting $\mathrm{T}$ cells. As mentioned above, it has been proposed that Vpx participates in the nuclear import of viral reverse transcription complexes (Fletcher et al., 1996; Pancio et al., 2000; Ueno et al., 2003; Belshan et al., 2006). Possibly in favor of such an additional role of $\mathrm{Vpx}$, some $\mathrm{Vpx}$ mutants have a more profound effect on MDDCs transduction with SIVmac lentiviral vectors than with HIV-1 (e.g., T17A, T28A, or GC86,87A Vpx mutants which fail to rescue SIVmac infection but improve HIV-1 infection by more than one order of magnitude Goujon et al., 2008; Table 1). Moreover, a Vpx mutant devoid of the C-terminus proline-rich 


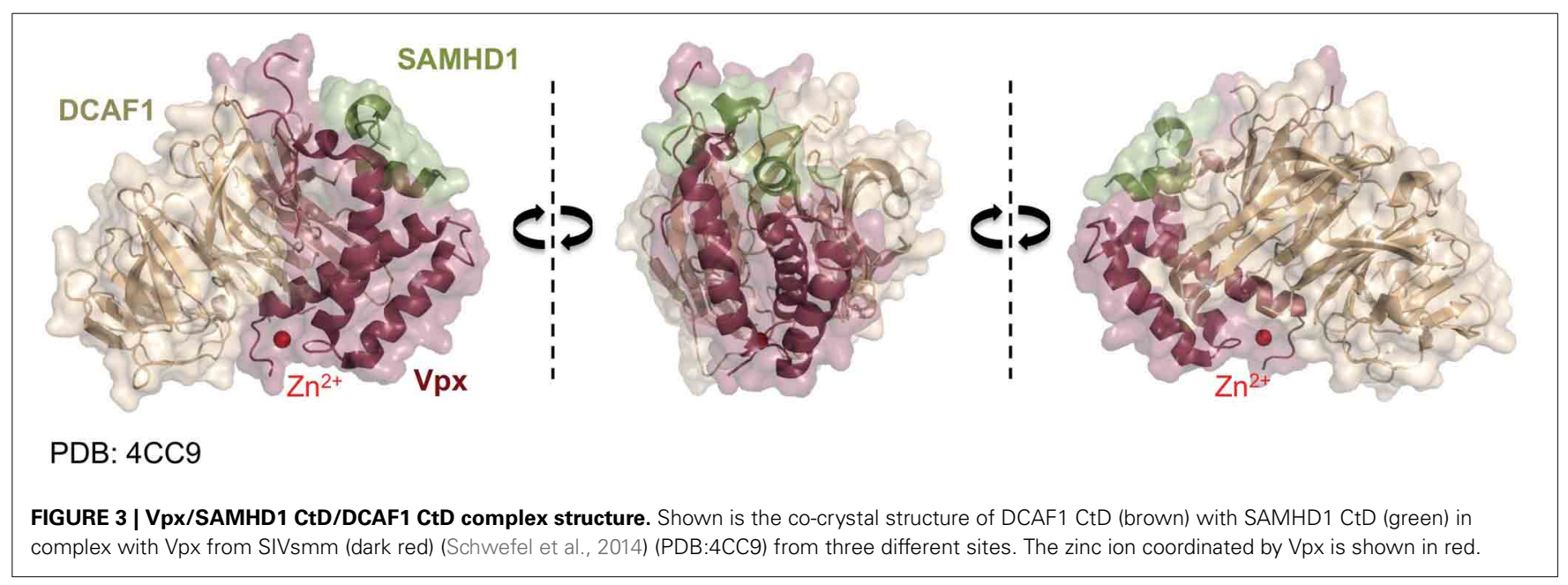

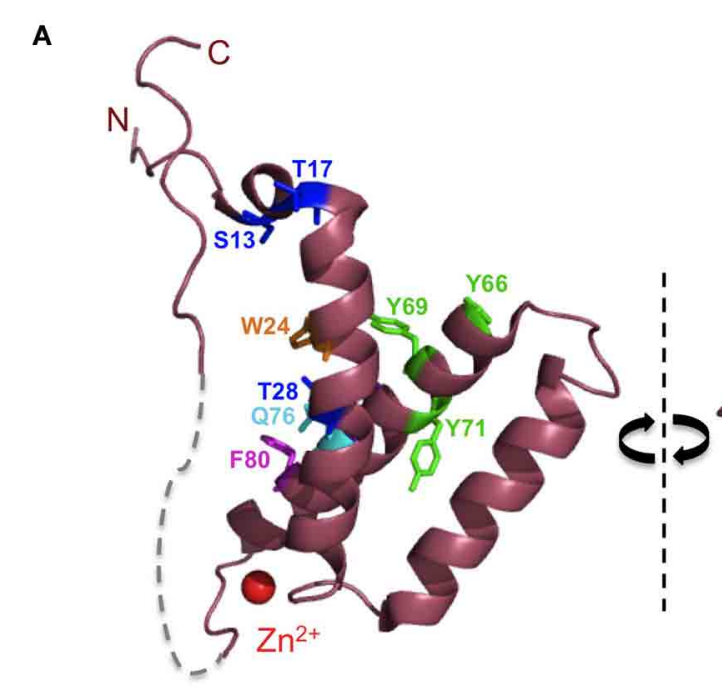

B

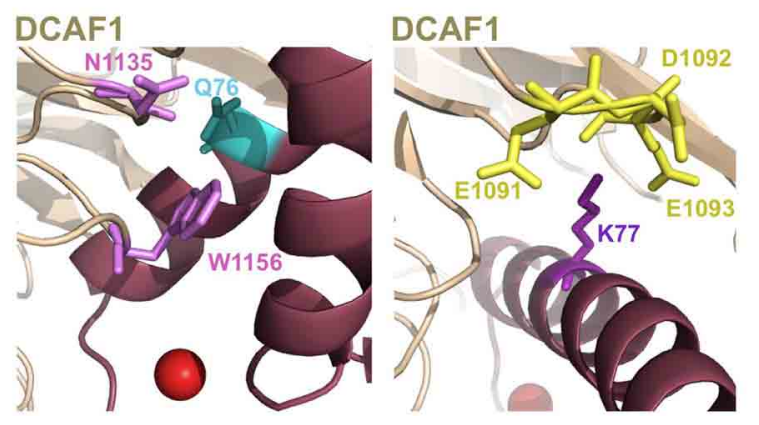

FIGURE $\mathbf{4}$ | Vpx structure. (A) Depicted is the structure of $V p x$ from SIVsmm as obtained in the co-crystallization with the C-terminus of DCAF1 and the C-terminus of SAMHD1 (both not shown) with critical amino acids highlighted (PDB:4CC9) (Schwefel et al., 2014). SIVsmm $V p x$ comprises an anti-parallel bundle of three helices, resembling the shape of a V. Vpx coordinates a zinc ion (red) with amino acids H39, H82, C87, and C89. The structure between Vpx residues 90-100 is absent (dashed gray line). (B) Critical aminoacid residues in the interface between DCAF1 and $V p x$ are highlighted. $V p x$ Q76 residue

\section{SAMHD1}
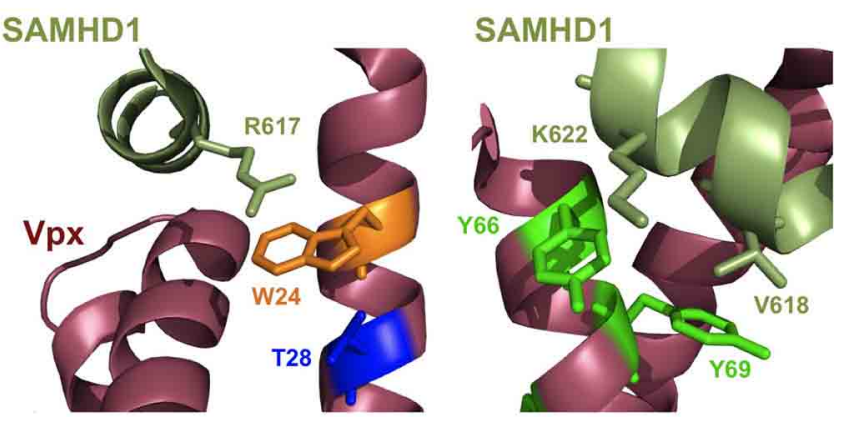

(cyan) may form hydrogen bonds with $\mathrm{N} 1135$ and W1156 residues (lilac) of DCAF1. The acidic region in DCAF1 comprising E1091, D1092 and E1093 residues (yellow) may interact with positively charged K77 residue (purple) of Vpx. (C) Critical amino acid residues for the interaction of SAMHD1 and $V p x$ are highlighted. SAMHD1 R617 residue (light green) stacks with the indole ring of Vpx W24 residue (orange). Vpx Y66 and Y69 residues (fluorescent green) are in close proximity to SAMHD1 K622 and V618 residues (light green), the latter being positively selected (Laguette et al., 2012). 
Table 1 | Phenotype of Vpx mutants.

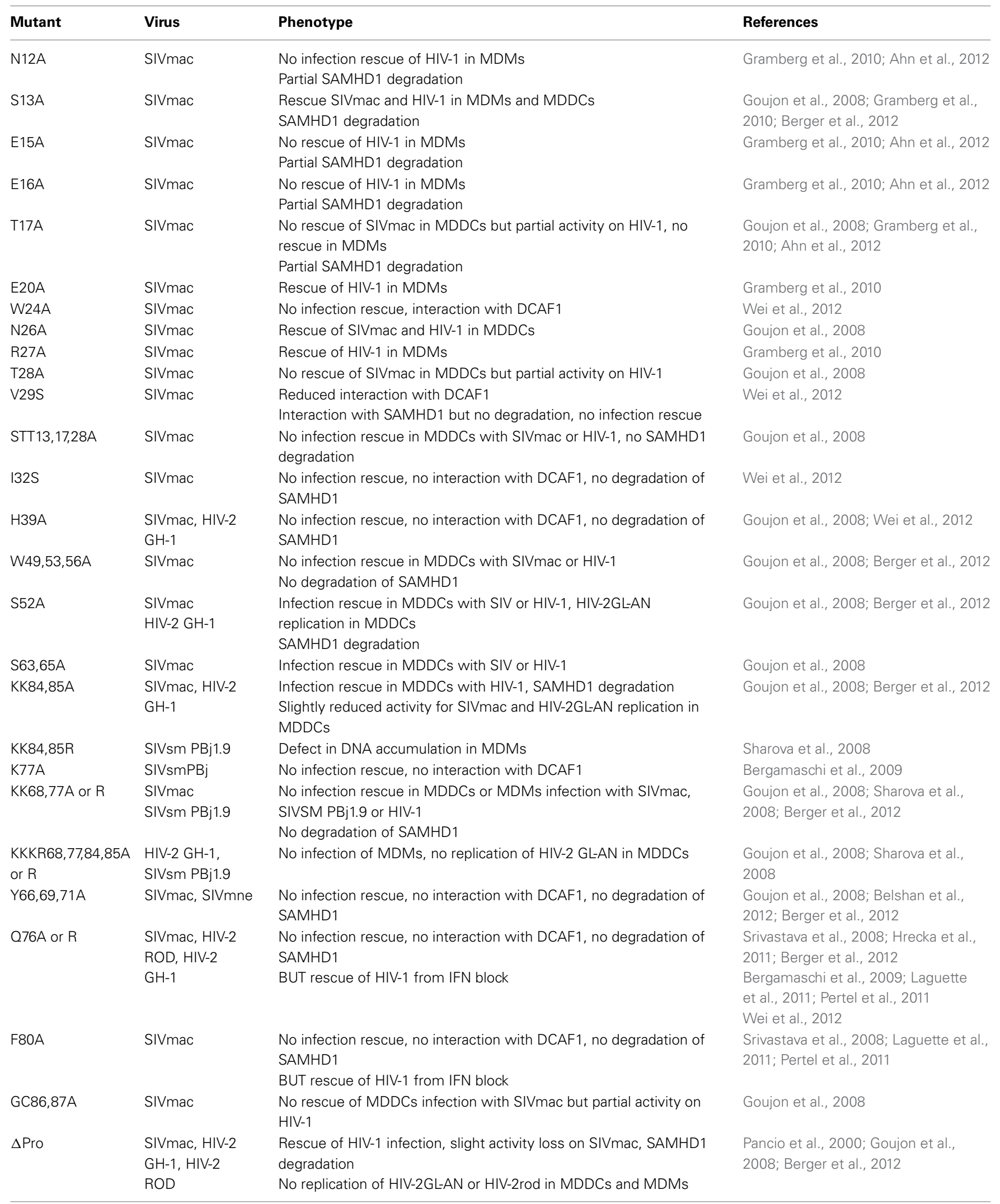

Non-exhaustive list of characterized Vpx mutants, summarizing their activity either in single-cycle infection with SIVmac and HIV-1 or in replication (in MDMs and/or $M D D C s)$, and their ability to interact with DCAF1 and SAMHD1, and to degrade the latter. 
region is still able to efficiently degrade SAMHD1 and to rescue HIV-1 in single-cycle infection of MDDCs (Goujon et al., 2008; Berger et al., 2012), but does not support HIV-2 replication in MDMs or MDDCs (Pancio et al., 2000; Goujon et al., 2008). Finally, Vpx was proposed to increase HIV-1 infectivity in a DCAF1-independent way both in IFN-treated MDDCs and in THP-1 cells, suggesting additional function(s) beyond SAMHD1 degradation (Goujon et al., 2008; Pertel et al., 2011; Reinhard et al., 2014).

\section{APOBEC3A ANTAGONISM?}

In addition to the well-documented interaction with SAMHD1, Vpx interacts with a member of the apolipoprotein B mRNAediting enzyme catalytic polypeptide-like 3 (APOBEC3) family of cytidine deaminases, namely APOBEC3A. APOBEC3 family members, exemplified by APOBEC $3 \mathrm{G}$, are potent restriction factors (reviewed in Malim and Bieniasz, 2012). HIV-1 prevents $A P O B E C 3 \mathrm{G} / \mathrm{F} / \mathrm{D} / \mathrm{H}$ action through proteasomal degradation induced by the Vif accessory protein. APOBEC3A affects various viruses and retroelements, such as human papilloma virus (Vartanian et al., 2008), adeno-associated virus (AAV) (Chen et al., 2006), and retrotransposons (Bogerd et al., 2006; Chen et al., 2006; Muckenfuss et al., 2006). Interestingly, APOBEC3A expression levels have been linked to myeloid cell restriction of HIV-1 infection (Peng et al., 2007). In line with this, APOBEC3A silencing increased the ability of HIV-1 to replicate in MDMs (Peng et al., 2007; Berger et al., 2011b). Two independent groups have shown that HIV-2/SIVsmm Vpx interacts with human APOBEC3A using co-immunoprecipitation of overexpressed proteins (Berger et al., 2010, 2011b). Both groups reported a decreased stability of APOBEC3A in the presence of Vpx. Similarly, wild-type SIVsmm replication led to slightly decreased APOBEC3A expression levels in monocytes compared to Vpx-deficient viruses (Berger et al., 2010). These data led to the hypothesis that Vpx might enhance infection of myeloid cells by inducing APOBEC3A degradation. However, the precise contribution of APOBEC3A to the resistance of HIV-1 infection in myeloid cells is difficult to evaluate, as SAMHD1 imposes a strong barrier to infection in these cells and is counteracted by Vpx. It would be crucial to separate, if possible, the two activities of $\mathrm{Vpx}$, for instance by identifying mutants of $\mathrm{Vpx}$ retaining the ability of interacting with APOBEC3A but not SAMHD1 (or vice versa), and evaluating their effect in myeloid cells.

\section{ANTAGONISM OF INTERFERON-INDUCED FACTOR(S)?}

It has been known for decades that type 1 IFN treatment potently decreases HIV-1 infection in some immortalized cell lines and in primary cells, such as MDMs and MDDCs (Kornbluth et al., 1989; Shirazi and Pitha, 1992; Baca-Regen et al., 1994; Cheney and McKnight, 2010; Goujon and Malim, 2010). Interestingly, this IFN-induced block is primarily exerted at the level of viral DNA accumulation (Shirazi and Pitha, 1993; Baca-Regen et al., 1994; Cheney and McKnight, 2010; Goujon and Malim, 2010), which is exactly the step at which SAMHD1 acts (Hrecka et al., 2011; Laguette et al., 2011). Strikingly, Vpx is still capable of enhancing HIV-1 infection in IFN-treated myeloid cells and the magnitude of the infectivity enhancement is increased in this context (Gramberg et al., 2010; Pertel et al., 2011; Goujon et al., 2013). SAMHD1 was reported to be IFN-inducible in some cell types, such as primary monocytes (Berger et al., 2011a). This suggested that SAMHD1 might be playing a role in the IFN block to HIV-1 infection. But neither SAMHD1 expression nor dNTP intracellular concentrations seem to be regulated by IFN treatment in MDMs or MDDCs (St Gelais et al., 2012; Dragin et al., 2013; Goujon et al., 2013). Furthermore, RNAi-mediated SAMHD1 silencing fails to rescue myeloid cell infection in the presence of IFN (Dragin et al., 2013; Goujon et al., 2013). In addition, Q76A Vpx mutant was still able to substantially rescue IFN-treated MDDC infection (Pertel et al., 2011; Reinhard et al., 2014), although it was unable to bind DCAF1 (Pertel et al., 2011) and induce SAMHD1 degradation (Hrecka et al., 2011). Whereas exogenous $\mathrm{dN}$ and SIVmac Vpx increased the accumulation of HIV-1 late reverse transcription products to the same extent in IFN-treated myeloid cells, Vpx had a higher impact on 2-LTR circle and proviral DNA formation (Reinhard et al., 2014). This feature was not observed with SIVmus and SIVdeb Vpr, despite their ability to degrade human SAMHD1 (Reinhard et al., 2014). Taken together, these data strongly suggest that HIV-2/SIVmac Vpx may be counteracting other myeloid specific, IFN-inducible anti-HIV-1 factor(s). It could be tempting to speculate that APOBEC3A may be one of these. Indeed, APOBEC3A is highly induced by IFN treatment in myeloid cells (Peng et al., 2006; Koning et al., 2009; Refsland et al., 2010) and Vpx is known to interact with APOBEC3A (Berger et al., 2010, 2011b). However, APOBEC3A was not degraded in the presence of $\mathrm{Vpx}$ VLPs following IFN treatment, contrary to SAMHD1, which is potently and rapidly degraded (Dragin et al., 2013). It remains possible that APOBEC3A is sequestered by $\mathrm{Vpx}$ rather than degraded in these conditions. Single-genome sequencing of viral DNA following HIV-1 infection of IFN-treated MDMs showed only infrequent editing and no sign of hypermutation, arguing against a major role for APOBEC3 proteins in the IFN block (Koning et al., 2011), though this does not exclude a deaminationindependent mechanism. Indeed, APOBEC3A is known to inhibit AAV via a deamination-independent mechanism (Narvaiza et al., 2009). Further work will be required to address the exact role of APOBEC3A in the IFN-induced block to HIV-1 infection, and to determine whether Vpx is counteracting additional IFN-induced anti-HIV-1 factors in myeloid cells. Of note, SIVmac lentiviral vectors are still sensitive to the antiviral action of IFN in human primary cells despite the presence of Vpx (Goujon and Malim, 2010; Reinhard et al., 2014). This may reflect the existence of species-specific IFN-induced genes to which SIVmac is sensitive in human cells contrary to HIV-1, and which are not counteracted by Vpx (Bitzegeio et al., 2013; Cordeil et al., 2013).

\section{ORIGIN AND EVOLUTION OF vpx}

The HIV-2/SIVsmm and SIVrcm/SIVmnd-2 lineages possess 2 homologous genes to HIV-1/SIVcpz $v p r$ : $v p r$ and $v p x$. The $v p r$ and $v p x$ genes are paralogs and are the result of complex duplication and/or recombination of their precursor throughout the diversification of primate lentiviruses. $v p r$ has most likely diverged through cross-species transmission, rather than co-evolution with their hosts following ancient infection. To 
illustrate this, Wertheim and Worobey studied mitochondrial sequences of several species of the African green monkey lineage and their SIVagm and have rejected the hypothesis of codivergence between SIVagm and its host. Their data support the hypothesis of a host-switching model following a geographical pattern of transmission (Wertheim and Worobey, 2007). Whilst $v p r$ is found in all lentiviral groups, $v p x$ is found only in two lineages: HIV-2/SIVsmm/SIVmac and SIVrcm/SIVmnd-2 (Beer et al., 2001; Hu et al., 2003). These viruses infect sooty mangabeys, macaques, mandrills and red-capped mangabeys, all of which belong to the same primate family.

The $v p x$ genes from the SIVrcm/SIVmnd-2 lineage are quite divergent from other $v p x$ genes (only about $30-40 \%$ identity at the amino acid level with Vpx from HIV-2/SIVsmm). Nonetheless, the monophyletic nature of the $v p x$ clade supports the hypothesis of a single event leading to the birth of $v p x$, rather than multiple events (Hu et al., 2003).

Two hypotheses have been proposed so far to explain the birth of $v p x$. It has first been suggested that $v p x$ arose by duplication of the $v p r$ ancestor (Tristem et al., 1990). This was supported by sequence similarity and by the fact that both genes are always adjacent to each other in the genome. Alternatively, because $v p x$ genes are closely related to $v p r$ from SIVagm.Sab (infecting African green monkeys) (Figure 5), it has been proposed by another group that a recombination event between SIVagm and SIVsmm could have led to the acquisition of SIVagm.Sab $v p r$ by the latter (Sharp et al., 1996; Hu et al., 2003). In agreement with this, recombination events between those two viruses have been shown before (Jin et al., 1994) and the habitats of African green monkeys and sooty mangabeys are partly overlapping. As the $\mathrm{Vpr}$ proteins of the HIV-2/SIVsmm/SIVmac and SIVrcm/SIVmnd-2 lineages are inactive against SAMHD1 (Lim et al., 2012) and as this function was acquired by the other $v p r$ genes before the birth of $v p x$, it seems likely that the former Vpr proteins were never able to antagonize SAMHD1. Therefore, the acquisition of $v p r$ from SIVagm would have been beneficial in this respect. Along these lines, it has been shown that the Vpr protein of SIVagm.Ver is able to antagonize a broad range of SAMHD1 proteins from primates (Lim et al., 2012). It is tempting to speculate that this may also be the case for SIVagm.Sab Vpr. This would favor the recombination (rather than the duplication) hypothesis, but would need to be assessed experimentally.

The rate of evolution of $v p x$ seems to be slower than that of $v p r$, as shown by shorter branch lengths in the $v p x$ lineage compared to the $v p r$ one (see the HIV-2/SIVsmm/SIVmac lineage in Figure 5) (Tristem et al., 1992). This could be explained by the fact that $v p x$ is overlapping with other genes to a greater degree than $v p r$, inducing a stronger constraint. In HIV-1 HXB2 for instance, 28\% of the $v p r$ gene is overlapping with other reading frames ( $v$ if and tat), whereas in HIV-2 BEN, 50\% of $v p x$ is overlapping (with $v p r$ ). A difference in selective pressures could also explain this disparity in evolution rate.

SIVcpz, which gave rise to the four known groups of HIV-1 after cross-species transmissions, is the result of recombination events between ancestors of SIVs from red-capped mangabeys and Cercopithecus species such as greater spot-nosed, mustached and mona monkeys (Bailes et al., 2003; Sharp and
Hahn, 2011). Each of these viruses has the ability to antagonize SAMHD1 from the species they target (SIVrcm with $v p x$ and SIVgsn/SIVmus/SIVmon with $v p r$ ) (Lim et al., 2012). However, this function was lost in SIVcpz (Etienne et al., 2013). As $v p x$ from SIVrcm was unable to antagonize chimpanzee SAMHD1 (Lim et al., 2012), the loss of $v p x$ might have had minimal consequences for the transmission of SIV to chimpanzees. It was hypothesized that the loss of $v p x-v p r$ remained intact-led to the reconstruction of vif by overprinting and the acquisition of full antagonism of APOBEC3 proteins from chimpanzee (Etienne et al., 2013). One might speculate that evolution of vif to fully counteract chimpanzee APOBEC3G was more important for SIVcpz than evolution of $v p x$ as a SAMHD1 antagonist.

Evolutionary analysis of SAMHD1 revealed strong signatures of positive selection in the $\mathrm{N}$ - and C-terminal parts of the protein (Laguette et al., 2012; Lim et al., 2012), suggesting that both termini may contribute to the interaction with Vpx. It has been shown recently that the separation of the two clades of $v p x$ (HIV-2/SIVsmm/SIVmac and SIVrcm/SIVmnd-2) correlates with the domain of SAMHD1 targeted by these $\mathrm{Vpx}$ proteins. $\mathrm{Vpx}$ proteins from the HIV-2/SIVsmm/SIVmac lineage recognize the C-terminal domain of SAMHD1 (Ahn et al., 2012; Fregoso et al., 2013), whereas Vpx proteins from the SIVrcm/SIVmnd-2 lineage interact with the N-terminal domain of SAMHD1 (Fregoso et al., 2013; Wei et al., 2014). The model presented to explain this involves the particular head-to-tail dimer conformation of SAMHD1 and a Vpx (or Vpr) protein binding to both N-terminal and C-terminal domains of SAMHD1 with a high affinity for one of these domains and a lower affinity for the other. When mutations occurring in SAMHD1 lead to a decreased affinity and escape from $\mathrm{Vpx}$, mutants of Vpx leading to higher affinity on the other domain might be selected (Fregoso et al., 2013). This evolutionary arms-race likely led to species specificity (Fregoso et al., 2013; Wei et al., 2014) and emphasizes the importance of the conservation of $\mathrm{Vpx}(\mathrm{Vpr})$ mediated SAMHD1 antagonism for lentiviruses. In agreement with this notion, SAMHD1 antagonism is actively maintained in natural infections, as exemplified by SIVagm $v p r$ adaptations to SAMHD1 polymorphisms found in the African green monkey population (Spragg and Emerman, 2013).

\section{HIV-1, A PANDEMIC VIRUS LACKING THE X-TRAORDINARY PROTEIN}

Although essential for certain primate lentiviruses, it is somewhat surprising that the ability to antagonize SAMHD1, as well as any other functions borne by $v p x$, were lost during the genesis of SIVcpz (Etienne et al., 2013). They were dispensable for the establishment of SIVcpz in chimpanzees and its cross-species transmission to humans, which gave rise to HIV-1. Whereas the other potential functions of $v p x$ might be provided by other genes in HIV-1/SIVcpz, it seems that HIV-1 does not need to antagonize SAMHD1 to replicate efficiently in humans. It is still not understood why SAMHD1 antagonism is crucial in some primate lentiviruses but dispensable in others. HIV-1 mainly targets cycling CD4 $+\mathrm{T}$ cells in which dNTP concentrations are high (Diamond et al., 2004), hence SAMHD1 antagonism may not be needed. Of note, HIV-1 


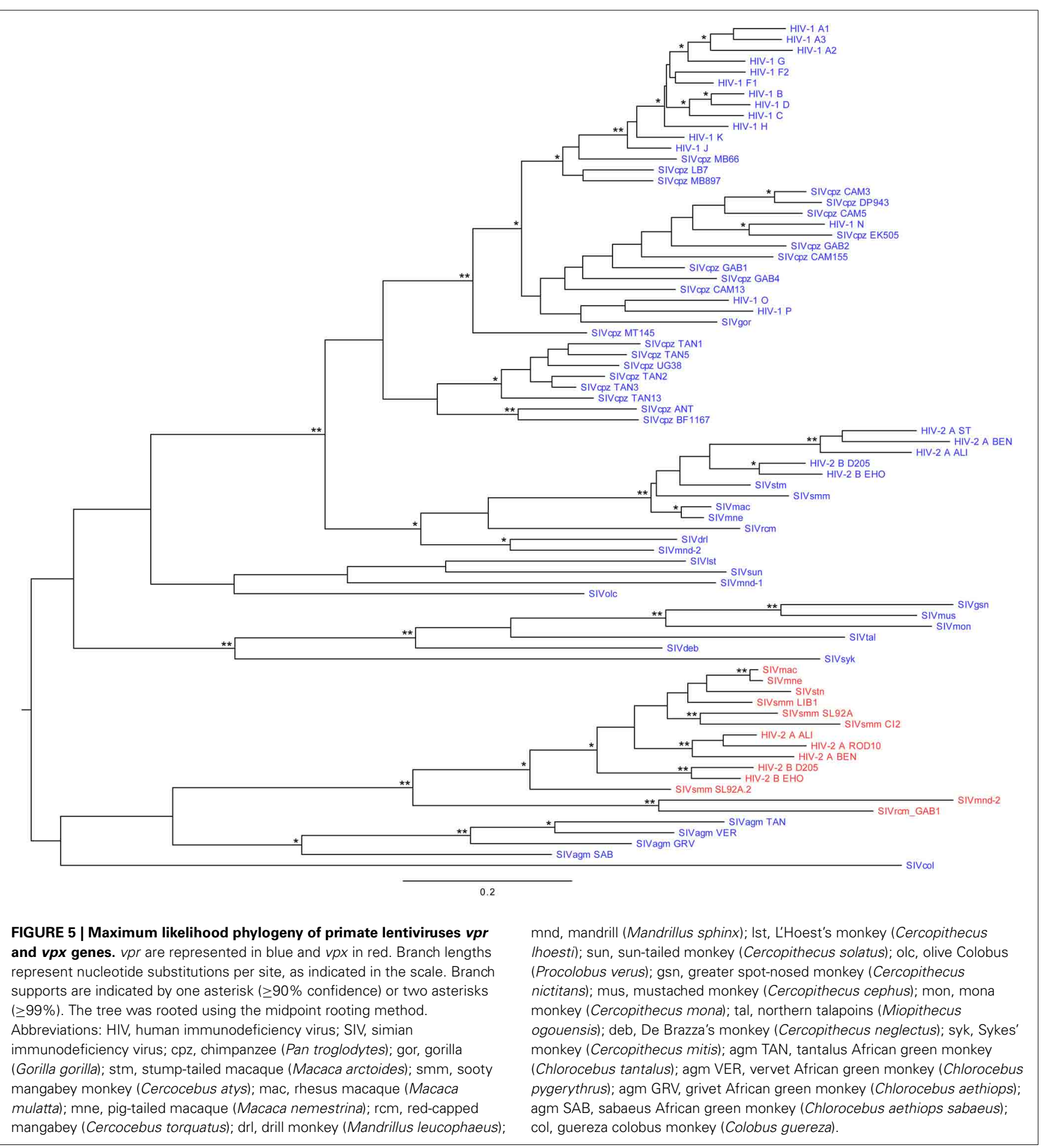

reverse transcriptase (RT) has a relatively high binding affinity for dNTPs, as compared to MLV (Weiss et al., 2004), which facilitates replication in cells with reduced dNTP content such as MDMs (Diamond et al., 2004). In line with this, HIV-1 RT mutant V148I, which has reduced dNTP binding affinity (Diamond et al., 2003, 2004), decreases HIV-1's ability to infect MDMs (Diamond et al., 2004; Lahouassa et al., 2012). Whether the RTs of HIV-2/SIVsmm have lower dNTP binding affinities than HIV-1 RT is currently unknown, but this could contribute to their dependence upon $v p x$. Alternatively viruses coding $v p x$ might rely more on myeloid cell infection to disseminate in vivo than the ones lacking this gene. Of note, most non-primate lentiviruses, such as maedi-visna or caprine arthritis-encephalitis virus, tend toward myeloid tropism (though the mechanisms 
used by these viruses to cope with low dNTP levels are currently unknown).

It would be of major interest to determine whether there is a connection between SAMHD1 degradation and an altered equilibrium between pathogen replication and host survival. Indeed primate lentiviruses able to antagonize SAMHD1 seem better tolerated by their natural hosts. This is exemplified by the difference observed in pathogenicity between HIV-1 and SIVmnd-1 compared to HIV-2, SIV smm and SIVmnd-2.

The in vitro infection of MDDCs with HIV-2 causes their activation through detection of viral cDNA by the DNA sensor cyclic GMP-APM synthase (cGAS) (Lahaye et al., 2013). In contrast, HIV-1 is normally unable to infect MDDCs and therefore does not activate them (Manel et al., 2010). However, HIV-1 can be artificially rendered able to infect DCs through the provision of $\mathrm{Vpx}$, leading to potent MDDC activation and secretion of cytokines including type 1 IFN (Manel et al., 2010; Lahaye et al., 2013). Under these artificial conditions HIV-1 seems to cause a stronger MDDC activation than HIV-2 (Yu et al., 2013). This strongly suggests that the absence of Vpx may actually be beneficial for HIV-1 as this would help avoiding infection and activation of DCs and therefore prevent initial innate (and then adaptive) immune responses detrimental to the virus. This would not be a unique example of a virus benefiting from limiting myeloid cell tropism. Indeed mosquito-borne North American eastern equine encephalitis virus (EEEV) is potently restricted by a specific miRNA in myeloid cells, whereas the sequence targeted by this miRNA is crucial for mosquito vector infection (Trobaugh et al., 2013). By limiting myeloid cell tropism and consequent innate immunity induction, the restriction directly promotes neurologic disease manifestations characteristic of EEEV infection in humans. In line with this, it is tempting to speculate that HIV-2 might be less pathogenic because it induces innate immune responses, possibly through myeloid cell infection. However, $v p x$ isolated from viremic and long-term aviremic HIV-2 infected individuals display similar abilities to antagonize SAMHD1 and enhance virus infection (Yu et al., 2013), presumably reflecting the complexity of viral pathogenesis.

Interestingly, there is some evidence that individuals presenting HIV-1/HIV-2 co-infections have better long-term outcomes and slower progression to AIDS, compared to HIV-1 monoinfected patients (Esbjornsson et al., 2012). HIV-2 co-infection seems to protect from HIV-1 pathogenesis for a certain period of time. It would be of high interest to understand the basis for this and whether Vpx plays a role in it.

\section{CONCLUSION}

$\mathrm{Vpx}$ is a protein uniquely encoded by the HIV-2/SIVsmm and SIVrcm/SIVmnd-2 lineages, which has the property to favor myeloid cell infection through inducing the degradation of SAMHD1. It is absolutely required for efficient HIV-2/SIVsmm viral replication in vivo, suggesting an important role of myeloid cells as target cells for these viruses and/or that additional roles, such as APOBEC3A antagonism, may be important. In addition, $\mathrm{Vpx}$ is able to render DCs more permissive to HIV-1 infection, and this may promote the induction of stronger innate immune responses. These major findings might open the way for the prospective development of novel HIV-1 vaccine and treatment strategies based on the use of $\mathrm{Vpx}$. In theory, treatment with a vaccine using Vpx-VLPs may stimulate immune responses, potentially leading to improved protection against (or control of) HIV-1.

\section{REFERENCES}

Accola, M. A., Bukovsky, A. A., Jones, M. S., and Gottlinger, H. G. (1999). A conserved dileucine-containing motif in p6(gag) governs the particle association of Vpx and Vpr of simian immunodeficiency viruses SIV(mac) and SIV(agm). J. Virol. 73, 9992-9999.

Ahn, J., Hao, C., Yan, J., Delucia, M., Mehrens, J., Wang, C., et al. (2012). HIV/simian immunodeficiency virus (SIV) accessory virulence factor Vpx loads the host cell restriction factor SAMHD1 onto the E3 ubiquitin ligase complex CRL4DCAF1. J. Biol. Chem. 287, 12550-12558. doi: 10.1074/jbc.M112. 340711

Akari, H., Sakuragi, J., Takebe, Y., Tomonaga, K., Kawamura, M., Fukasawa, M., et al. (1992). Biological characterization of human immunodeficiency virus type 1 and type 2 mutants in human peripheral blood mononuclear cells. Arch. Virol. 123, 157-167. doi: 10.1007/BF01317146

Angers, S., Li, T., Yi, X., Maccoss, M. J., Moon, R. T., and Zheng, N. (2006). Molecular architecture and assembly of the DDB1-CUL4A ubiquitin ligase machinery. Nature 443, 590-593. doi: 10.1038/nature05175

Baca-Regen, L., Heinzinger, N., Stevenson, M., and Gendelman, H. E. (1994). Alpha interferon-induced antiretroviral activities: restriction of viral nucleic acid synthesis and progeny virion production in human immunodeficiency virus type 1-infected monocytes. J. Virol. 68, 7559-7565.

Bailes, E., Gao, F., Bibollet-Ruche, F., Courgnaud, V., Peeters, M., Marx, P. A., et al. (2003). Hybrid origin of SIV in chimpanzees. Science 300, 1713. doi: $10.1126 /$ science. 1080657

Baldauf, H. M., Pan, X., Erikson, E., Schmidt, S., Daddacha, W., Burggraf, M., et al. (2012). SAMHD1 restricts HIV-1 infection in resting CD4(+) T cells. Nat. Med. 18, 1682-1689. doi: 10.1038/nm.2964

Beck-Engeser, G. B., Eilat, D., and Wabl, M. (2011). An autoimmune disease prevented by anti-retroviral drugs. Retrovirology 8, 91. doi: 10.1186/17424690-8-91

Beer, B. E., Foley, B. T., Kuiken, C. L., Tooze, Z., Goeken, R. M., Brown, C. R., et al. (2001). Characterization of novel simian immunodeficiency viruses from red-capped mangabeys from Nigeria (SIVrcmNG409 and -NG411). J. Virol. 75, 12014-12027. doi: 10.1128/JVI.75.24.12014-12027.2001

Behrendt, R., Schumann, T., Gerbaulet, A., Nguyen, L. A., Schubert, N., Alexopoulou, D., et al. (2013). Mouse SAMHD1 has antiretroviral activity and suppresses a spontaneous cell-intrinsic antiviral response. Cell Rep. 4, 689-696. doi: 10.1016/j.celrep.2013.07.037

Beloglazova, N., Flick, R., Tchigvintsev, A., Brown, G., Popovic, A., Nocek, B., et al. (2013). Nuclease activity of the human SAMHD1 protein implicated in the Aicardi-Goutieres syndrome and HIV-1 restriction. J. Biol. Chem. 288, 8101-8110. doi: 10.1074/jbc.M112.431148

Belshan, M., Kimata, J. T., Brown, C., Cheng, X., McCulley, A., Larsen, A., et al. (2012). Vpx is critical for SIVmne infection of pigtail macaques. Retrovirology 9, 32. doi: 10.1186/1742-4690-9-32

Belshan, M., Mahnke, L. A., and Ratner, L. (2006). Conserved amino acids of the human immunodeficiency virus type $2 \mathrm{Vpx}$ nuclear localization signal are critical for nuclear targeting of the viral preintegration complex in non-dividing cells. Virology 346, 118-126. doi: 10.1016/j.virol.2005.10.036

Belshan, M., and Ratner, L. (2003). Identification of the nuclear localization signal of human immunodeficiency virus type 2 Vpx. Virology 311, 7-15. doi: 10.1016/S0042-6822(03)00093-X

Bergamaschi, A., Ayinde, D., David, A., Le Rouzic, E., Morel, M., Collin, G., et al. (2009). The human immunodeficiency virus type $2 \mathrm{Vpx}$ protein usurps the CUL4A-DDB1 DCAF1 ubiquitin ligase to overcome a postentry block in macrophage infection. J. Virol. 83, 4854-4860. doi: 10.1128/JVI.00187-09

Berger, A., Munk, C., Schweizer, M., Cichutek, K., Schule, S., and Flory, E. (2010). Interaction of $\mathrm{Vpx}$ and apolipoprotein $\mathrm{B}$ mRNA-editing catalytic polypeptide 3 family member A (APOBEC3A) correlates with efficient lentivirus infection of monocytes. J. Biol. Chem. 285, 12248-12254. doi: 10.1074/jbc.M109.090977

Berger, A., Sommer, A. F., Zwarg, J., Hamdorf, M., Welzel, K., Esly, N., et al. (2011a). SAMHD1-deficient CD14+ cells from individuals with Aicardi-Goutieres 
syndrome are highly susceptible to HIV-1 infection. PLoS Pathog. 7:e1002425. doi: 10.1371/journal.ppat. 1002425

Berger, G., Durand, S., Fargier, G., Nguyen, X. N., Cordeil, S., Bouaziz, S., et al. (2011b). APOBEC3A is a specific inhibitor of the early phases of HIV-1 infection in myeloid cells. PLoS Pathog. 7:e1002221. doi: 10.1371/journal.ppat.1002221

Berger, G., Turpin, J., Cordeil, S., Tartour, K., Nguyen, X. N., Mahieux, R., et al. (2012). Functional analysis of the relationship between Vpx and the restriction factor SAMHD1. J. Biol. Chem. 287, 41210-41217. doi: 10.1074/jbc.M112.403816

Bitzegeio, J., Sampias, M., Bieniasz, P. D., and Hatziioannou, T. (2013). Adaptation to the interferon-induced antiviral state by human and simian immunodeficiency viruses. J. Virol. 87, 3549-3560. doi: 10.1128/JVI.03219-12

Bloch, N., O'Brien, M., Norton, T. D., Polsky, S. B., Bhardwaj, N., and Landau, N. R. (2014). HIV Type 1 infection of plasmacytoid and myeloid dendritic cells is restricted by high levels of SAMHD1 and cannot be counteracted by Vpx. AIDS Res. Hum. Retroviruses 30, 195-203. doi: 10.1089/AID.2013.0119

Bogerd, H. P., Wiegand, H. L., Doehle, B. P., Lueders, K. K., and Cullen, B. R. (2006). APOBEC $3 \mathrm{~A}$ and APOBEC3B are potent inhibitors of LTR-retrotransposon function in human cells. Nucleic Acids Res. 34, 89-95. doi: 10.1093/nar/gkj416

Brandariz-Nunez, A., Valle-Casuso, J. C., White, T. E., Laguette, N., Benkirane, M., Brojatsch, J., et al. (2012). Role of SAMHD1 nuclear localization in restriction of HIV-1 and SIVmac. Retrovirology 9, 49. doi: 10.1186/1742-4690-9-49

Chahwan, C., and Chahwan, R. (2012). Aicardi-Goutieres syndrome: from patients to genes and beyond. Clin. Genet. 81, 413-420. doi: 10.1111/j.13990004.2011.01825.x

Chen, H., Lilley, C. E., Yu, Q., Lee, D. V., Chou, J., Narvaiza, I., et al. (2006). APOBEC3A is a potent inhibitor of adeno-associated virus and retrotransposons. Curr. Biol. 16, 480-485. doi: 10.1016/j.cub.2006.01.031

Cheney, K. M., and McKnight, A. (2010). Interferon-alpha mediates restriction of human immunodeficiency virus type-1 replication in primary human macrophages at an early stage of replication. PLOS ONE 5:e13521. doi: 10.1371/journal.pone.0013521

Cordeil, S., Nguyen, X. N., Berger, G., Durand, S., Ainouze, M., and Cimarelli, A. (2013). Evidence for a different susceptibility of primate lentiviruses to type I interferons. J. Virol. 87, 2587-2596. doi: 10.1128/JVI.02553-12

Cribier, A., Descours, B., Valadao, A. L., Laguette, N., and Benkirane, M. (2013). Phosphorylation of SAMHD1 by cyclin A2/CDK1 regulates its restriction activity toward HIV-1. Cell Rep. 3, 1036-1043. doi: 10.1016/j.celrep.2013.03.017

Crow, Y. J., Hayward, B. E., Parmar, R., Robins, P., Leitch, A., Ali, M., et al. (2006). Mutations in the gene encoding the $3^{\prime}-5^{\prime}$ DNA exonuclease TREX1 cause Aicardi-Goutieres syndrome at the AGS1 locus. Nat. Genet. 38, 917-920. doi: $10.1038 / \mathrm{ng} 1845$

Delucia, M., Mehrens, J., Wu, Y., and Ahn, J. (2013). HIV-2 and SIVmac accessory virulence factor Vpx down-regulates SAMHD1 enzyme catalysis prior to proteasome-dependent degradation. J. Biol. Chem. 288, 19116-19126. doi: 10.1074/jbc.M113.469007

Depienne, C., Roques, P., Creminon, C., Fritsch, L., Casseron, R., Dormont, D., et al. (2000). Cellular distribution and karyophilic properties of matrix, integrase, and $\mathrm{Vpr}$ proteins from the human and simian immunodeficiency viruses. Exp. Cell Res. 260, 387-395. doi: 10.1006/excr.2000.5016

Descours, B., Cribier, A., Chable-Bessia, C., Ayinde, D., Rice, G., Crow, Y., et al. (2012). SAMHD1 restricts HIV-1 reverse transcription in quiescent CD4(+) T-cells. Retrovirology 9, 87. doi: 10.1186/1742-4690-9-87

Diamond, T. L., Roshal, M., Jamburuthugoda, V. K., Reynolds, H. M., Merriam, A. R., Lee, K. Y., et al. (2004). Macrophage tropism of HIV-1 depends on efficient cellular dNTP utilization by reverse transcriptase. J. Biol. Chem. 279, 51545-51553. doi: 10.1074/jbc.M408573200

Diamond, T. L., Souroullas, G., Weiss, K. K., Lee, K. Y., Bambara, R. A., Dewhurst, S., et al. (2003). Mechanistic understanding of an altered fidelity simian immunodeficiency virus reverse transcriptase mutation, V148I, identified in a pig-tailed macaque. J. Biol. Chem. 278, 29913-29924. doi: 10.1074/jbc.M2117 54200

Dragin, L., Nguyen, L. A., Lahouassa, H., Sourisce, A., Kim, B., Ramirez, B. C., et al. (2013). Interferon block to HIV-1 transduction in macrophages despite SAMHD1 degradation and high deoxynucleoside triphosphates supply. Retrovirology 10, 30. doi: 10.1186/1742-4690-10-30

Esbjornsson, J., Mansson, F., Kvist, A., Isberg, P. E., Nowroozalizadeh, S., Biague, A. J., et al. (2012). Inhibition of HIV-1 disease progression by contemporaneous HIV-2 infection. N. Engl. J. Med. 367, 224-232. doi: 10.1056/NEJMoa1113244
Etienne, L., Hahn, B. H., Sharp, P. M., Matsen, F. A., and Emerman, M. (2013). Gene loss and adaptation to hominids underlie the ancient origin of HIV-1. Cell Host Microbe 14, 85-92. doi: 10.1016/j.chom.2013.06.002

Fletcher, T. M. 3rd., Brichacek, B., Sharova, N., Newman, M. A., Stivahtis, G., Sharp, P. M., et al. (1996). Nuclear import and cell cycle arrest functions of the HIV$1 \mathrm{Vpr}$ protein are encoded by two separate genes in HIV-2/SIV(SM). EMBO J. $15,6155-6165$.

Franchini, G., Rusche, J. R., O'Keeffe, T. J., and Wong-Staal, F. (1988). The human immunodeficiency virus type 2 (HIV-2) contains a novel gene encoding a $16 \mathrm{kD}$ protein associated with mature virions. AIDS Res. Hum. Retroviruses 4, 243-250. doi: 10.1089/aid.1988.4.243

Fregoso, O. I., Ahn, J., Wang, C., Mehrens, J., Skowronski, J., and Emerman, M. (2013). Evolutionary toggling of $\mathrm{Vpx} / \mathrm{Vpr}$ specificity results in divergent recognition of the restriction factor SAMHD1. PLoS Pathog. 9:e1003496. doi: 10.1371/journal.ppat.1003496

Fujita, M., Otsuka, M., Miyoshi, M., Khamsri, B., Nomaguchi, M., and Adachi, A. (2008). Vpx is critical for reverse transcription of the human immunodeficiency virus type 2 genome in macrophages. J. Virol. 82, 7752-7756. doi: 10.1128/JVI.01003-07

Gibbs, J. S., Lackner, A. A., Lang, S. M., Simon, M. A., Sehgal, P. K., Daniel, M. D. et al. (1995). Progression to AIDS in the absence of a gene for vpr or vpx. J. Virol. 69, 2378-2383.

Gibbs, J. S., Regier, D. A., and Desrosiers, R. C. (1994). Construction and in vitro properties of SIVmac mutants with deletions in "nonessential" genes. AIDS Res. Hum. Retroviruses 10, 607-616. doi: 10.1089/aid.1994.10.607

Goldstone, D. C., Ennis-Adeniran, V., Hedden, J. J., Groom, H. C., Rice, G. I., Christodoulou, E., et al. (2011). HIV-1 restriction factor SAMHD1 is a deoxynucleoside triphosphate triphosphohydrolase. Nature 480, 379-382. doi: 10.1038 /nature10623

Goncalves, A., Karayel, E., Rice, G. I., Bennett, K. L., Crow, Y. J., Superti-Furga, G., et al. (2012). SAMHD1 is a nucleic-acid binding protein that is mislocalized due to aicardi-goutieres syndrome-associated mutations. Hum. Mutat. 33, 1116-1122. doi: 10.1002/humu.22087

Goujon, C., Arfi, V., Pertel, T., Luban, J., Lienard, J., Rigal, D., et al. (2008). Characterization of simian immunodeficiency virus SIVSM/human immunodeficiency virus type $2 \mathrm{Vpx}$ function in human myeloid cells. J. Virol. 82 , 12335-12345. doi: 10.1128/JVI.01181-08

Goujon, C., Jarrosson-Wuilleme, L., Bernaud, J., Rigal, D., Darlix, J. L., and Cimarelli, A. (2006). With a little help from a friend: increasing HIV transduction of monocyte-derived dendritic cells with virion-like particles of SIV(MAC). Gene Ther. 13, 991-994. doi: 10.1038/sj.gt.3302753

Goujon, C., and Malim, M. H. (2010). Characterization of the alpha interferoninduced postentry block to HIV-1 infection in primary human macrophages and T cells. J. Virol. 84, 9254-9266. doi: 10.1128/JVI.00854-10

Goujon, C., Riviere, L., Jarrosson-Wuilleme, L., Bernaud, J., Rigal, D., Darlix, J. L., et al. (2007). SIVSM/HIV-2 Vpx proteins promote retroviral escape from a proteasome-dependent restriction pathway present in human dendritic cells. Retrovirology 4, 2. doi: 10.1186/1742-4690-4-2

Goujon, C., Schaller, T., Galao, R. P., Amie, S. M., Kim, B., Olivieri, K., et al. (2013). Evidence for IFNalpha-induced, SAMHD1-independent inhibitors of early HIV-1 infection. Retrovirology 10, 23. doi: 10.1186/1742-4690-10-23

Gramberg, T., Kahle, T., Bloch, N., Wittmann, S., Mullers, E., Daddacha, W., et al. (2013). Restriction of diverse retroviruses by SAMHD1. Retrovirology 10, 26. doi: $10.1186 / 1742-4690-10-26$

Gramberg, T., Sunseri, N., and Landau, N. R. (2010). Evidence for an activation domain at the amino terminus of simian immunodeficiency virus Vpx. J. Virol. 84, 1387-1396. doi: 10.1128/JVI.01437-09

Guo, H., Wei, W., Wei, Z., Liu, X., Evans, S. L., Yang, W., et al. (2013). Identification of critical regions in human SAMHD1 required for nuclear localization and Vpx-mediated degradation. PLoS ONE 8:e66201. doi: 10.1371/journal.pone.0066201

Guyader, M., Emerman, M., Montagnier, L., and Peden, K. (1989). VPX mutants of HIV-2 are infectious in established cell lines but display a severe defect in peripheral blood lymphocytes. EMBO J. 8, 1169-1175.

Henderson, L. E., Sowder, R. C., Copeland, T. D., Benveniste, R. E., and Oroszlan, S. (1988). Isolation and characterization of a novel protein (X-ORF product) from SIV and HIV-2. Science 241, 199-201. doi: 10.1126/science.3388031

Hirsch, V. M., Sharkey, M. E., Brown, C. R., Brichacek, B., Goldstein, S., Wakefield, J., et al. (1998). Vpx is required for dissemination and pathogenesis of SIV(SM) 
PBj: evidence of macrophage-dependent viral amplification. Nat. Med. 4, 1401-1408. doi: 10.1038/3992

Hofmann, H., Logue, E. C., Bloch, N., Daddacha, W., Polsky, S. B., Schultz, M. L., et al. (2012). The Vpx lentiviral accessory protein targets SAMHD1 for degradation in the nucleus. J. Virol. 86, 12552-12560. doi: 10.1128/JVI.01657-12

Hofmann, H., Norton, T. D., Schultz, M. L., Polsky, S. B., Sunseri, N., and Landau, N. R. (2013). Inhibition of CUL4A neddylation causes a reversible block to SAMHD1-mediated restriction of HIV-1. J. Virol. 87, 11741-11750. doi: 10.1128/JVI.02002-13

Hollenbaugh, J. A., Gee, P., Baker, J., Daly, M. B., Amie, S. M., Tate, J., et al. (2013). Host factor SAMHD1 restricts DNA viruses in non-dividing myeloid cells. PLoS Pathog. 9:e1003481. doi: 10.1371/journal.ppat.1003481

Hrecka, K., Hao, C., Gierszewska, M., Swanson, S. K., Kesik-Brodacka, M., Srivastava, S., et al. (2011). Vpx relieves inhibition of HIV-1 infection of macrophages mediated by the SAMHD1 protein. Nature 474, 658-661. doi: 10.1038/nature10195

Hu, J., Switzer, W. M., Foley, B. T., Robertson, D. L., Goeken, R. M., Korber, B. T., et al. (2003). Characterization and comparison of recombinant simian immunodeficiency virus from drill (Mandrillus leucophaeus) and mandrill (Mandrillus sphinx) isolates. J. Virol. 77, 4867-4880. doi: 10.1128/JVI.77.8.4867-48 80.2003

Hu, W., Vander Heyden, N., and Ratner, L. (1989). Analysis of the function of viral protein X (VPX) of HIV-2. Virology 173, 624-630. doi: 10.1016/00426822(89)90574-6

Ji, X., Wu, Y., Yan, J., Mehrens, J., Yang, H., Delucia, M., et al. (2013). Mechanism of allosteric activation of SAMHD1 by dGTP. Nat. Struct. Mol. Biol. 20, 1304-1309. doi: $10.1038 / \mathrm{nsmb} .2692$

Jin, M. J., Hui, H., Robertson, D. L., Muller, M. C., Barre-Sinoussi, F., Hirsch, V. M., et al. (1994). Mosaic genome structure of simian immunodeficiency virus from west African green monkeys. EMBO J. 13, 2935-2947.

Kappes, J. C., Conway, J. A., Lee, S. W., Shaw, G. M., and Hahn, B. H. (1991). Human immunodeficiency virus type 2 vpx protein augments viral infectivity. Virology 184, 197-209. doi: 10.1016/0042-6822(91)90836-Z

Kappes, J. C., Morrow, C. D., Lee, S. W., Jameson, B. A., Kent, S. B., Hood, L. E., et al. (1988). Identification of a novel retroviral gene unique to human immunodeficiency virus type 2 and simian immunodeficiency virus SIVMAC. J. Virol. 62, 3501-3505.

Kaushik, R., Zhu, X., Stranska, R., Wu, Y., and Stevenson, M. (2009). A cellular restriction dictates the permissivity of nondividing monocytes/macrophages to lentivirus and gammaretrovirus infection. Cell Host Microbe 6, 68-80. doi: 10.1016/j.chom.2009.05.022

Kawamura, M., Sakai, H., and Adachi, A. (1994). Human immunodeficiency virus $\mathrm{Vpx}$ is required for the early phase of replication in peripheral blood mononuclear cells. Microbiol. Immunol. 38, 871-878. doi: 10.1111/j.13480421.1994.tb02140.x

Kewalramani, V. N., and Emerman, M. (1996). Vpx association with mature core structures of HIV-2. Virology 218, 159-168. doi: 10.1006/viro.1996.0176

Kim, E. T., White, T. E., Brandariz-Nunez, A., Diaz-Griffero, F., and Weitzman, M. D. (2013). SAMHD1 restricts herpes simplex virus 1 in macrophages by limiting DNA replication. J. Virol. 87, 12949-12956. doi: 10.1128/JVI.02291-13

Koning, F. A., Goujon, C., Bauby, H., and Malim, M. H. (2011). Target cell-mediated editing of HIV-1 cDNA by APOBEC3 proteins in human macrophages. J. Virol. 85, 13448-13452. doi: 10.1128/JVI.00775-11

Koning, F. A., Newman, E. N., Kim, E. Y., Kunstman, K. J., Wolinsky, S. M., and Malim, M. H. (2009). Defining APOBEC3 expression patterns in human tissues and hematopoietic cell subsets. J. Virol. 83, 9474-9485. doi: 10.1128/JVI.0 1089-09

Korin, Y. D., and Zack, J. A. (1999). Nonproductive human immunodeficiency virus type 1 infection in nucleoside-treated G0 lymphocytes. J. Virol. 73, 6526-6532.

Kornbluth, R. S., Oh, P. S., Munis, J. R., Cleveland, P. H., and Richman, D. D. (1989). Interferons and bacterial lipopolysaccharide protect macrophages from productive infection by human immunodeficiency virus in vitro. J. Exp. Med. 169, 1137-1151. doi: 10.1084/jem.169.3.1137

Laguette, N., Rahm, N., Sobhian, B., Chable-Bessia, C., Munch, J., Snoeck, J., et al. (2012). Evolutionary and functional analyses of the interaction between the myeloid restriction factor SAMHD1 and the lentiviral Vpx protein. Cell Host Microbe 11, 205-217. doi: 10.1016/j.chom.2012.01.007

Laguette, N., Sobhian, B., Casartelli, N., Ringeard, M., Chable-Bessia, C., Segeral, E., et al. (2011). SAMHD1 is the dendritic- and myeloid-cell-specific
HIV-1 restriction factor counteracted by Vpx. Nature 474, 654-657. doi: 10.1038 /nature 10117

Lahaye, X., Satoh, T., Gentili, M., Cerboni, S., Conrad, C., Hurbain, I., et al. (2013). The Capsids of HIV-1 and HIV-2 determine immune detection of the viral cDNA by the innate sensor cGAS in dendritic cells. Immunity 39, 1132-1142. doi: 10.1016/j.immuni.2013.11.002

Lahouassa, H., Daddacha, W., Hofmann, H., Ayinde, D., Logue, E. C., Dragin, L., et al. (2012). SAMHD1 restricts the replication of human immunodeficiency virus type 1 by depleting the intracellular pool of deoxynucleoside triphosphates. Nat. Immunol. 13, 223-228. doi: 10.1038/ni.2236

Le Rouzic, E., Belaidouni, N., Estrabaud, E., Morel, M., Rain, J. C., Transy, C., et al. (2007). HIV1 Vpr arrests the cell cycle by recruiting DCAF1/VprBP, a receptor of the Cul4-DDB1 ubiquitin ligase. Cell Cycle 6, 182-188. doi: $10.4161 /$ cc.6.2.3732

Lewis, P. F., and Emerman, M. (1994). Passage through mitosis is required for oncoretroviruses but not for the human immunodeficiency virus. J. Virol. 68, 510-516.

Li, N., Zhang, W., and Cao, X. (2000). Identification of human homologue of mouse IFN-gamma induced protein from human dendritic cells. Immunol. Lett. 74, 221-224. doi: 10.1016/S0165-2478(00)00276-5

Liao, W., Bao, Z., Cheng, C., Mok, Y. K., and Wong, W. S. (2008). Dendritic cellderived interferon-gamma-induced protein mediates tumor necrosis factoralpha stimulation of human lung fibroblasts. Proteomics 8, 2640-2650. doi: 10.1002/pmic.200700954

Lim, E. S., Fregoso, O. I., McCoy, C. O., Matsen, F. A., Malik, H. S., and Emerman, M. (2012). The ability of primate lentiviruses to degrade the monocyte restriction factor SAMHD1 preceded the birth of the viral accessory protein Vpx. Cell Host Microbe 11, 194-204. doi: 10.1016/j.chom.2012. 01.004

Mahalingam, S., Van Tine, B., Santiago, M. L., Gao, F., Shaw, G. M., and Hahn, B. H. (2001). Functional analysis of the simian immunodeficiency virus Vpx protein: identification of packaging determinants and a novel nuclear targeting domain. J. Virol. 75, 362-374. doi: 10.1128/JVI.75.1.362374.2001

Malim, M. H., and Bieniasz, P. D. (2012). HIV restriction factors and mechanisms of evasion. Cold Spring Harb. Perspect. Med. 2:a006940. doi: 10.1101/cshperspect.a006940

Manel, N., Hogstad, B., Wang, Y., Levy, D. E., Unutmaz, D., and Littman, D. R. (2010). A cryptic sensor for HIV-1 activates antiviral innate immunity in dendritic cells. Nature 467, 214-217. doi: 10.1038/nature09337

Mangeot, P. E., Duperrier, K., Negre, D., Boson, B., Rigal, D., Cosset, F. L., et al. (2002). High levels of transduction of human dendritic cells with optimized SIV vectors. Mol. Ther. 5, 283-290. doi: 10.1006/mthe.2002.0541

Muckenfuss, H., Hamdorf, M., Held, U., Perkovic, M., Lower, J., Cichutek, K., et al. (2006). APOBEC3 proteins inhibit human LINE-1 retrotransposition. J. Biol. Chem. 281, 22161-22172. doi: 10.1074/jbc.M601716200

Narvaiza, I., Linfesty, D. C., Greener, B. N., Hakata, Y., Pintel, D. J., Logue, E., et al. (2009). Deaminase-independent inhibition of parvoviruses by the APOBEC3A cytidine deaminase. PLoS Pathog. 5:e1000439. doi: 10.1371/journal.ppat.1000439

Neil, S., Martin, F., Ikeda, Y., and Collins, M. (2001). Postentry restriction to human immunodeficiency virus-based vector transduction in human monocytes. J. Virol. 75, 5448-5456. doi: 10.1128/JVI.75.12.5448-5456.2001

Pan, X., Baldauf, H. M., Keppler, O. T., and Fackler, O. T. (2013). Restrictions to HIV-1 replication in resting CD4+ T lymphocytes. Cell Res. 23, 876-885. doi: 10.1038/cr.2013.74

Pancio, H. A., Vander Heyden, N., and Ratner, L. (2000). The C-terminal prolinerich tail of human immunodeficiency virus type $2 \mathrm{Vpx}$ is necessary for nuclear localization of the viral preintegration complex in nondividing cells. J. Virol. 74, 6162-6167. doi: 10.1128/JVI.74.13.6162-6167.2000

Park, I. W., and Sodroski, J. (1995). Functional analysis of the vpx, vpr, and nef genes of simian immunodeficiency virus. J. Acquir. Immune Defic. Syndr. Hum. Retrovirol. 8, 335-344. doi: 10.1097/00042560-199504000-00003

Peng, G., Greenwell-Wild, T., Nares, S., Jin, W., Lei, K. J., Rangel, Z. G., et al. (2007). Myeloid differentiation and susceptibility to HIV-1 are linked to APOBEC3 expression. Blood 110, 393-400. doi: 10.1182/blood-2006-10051763

Peng, G., Lei, K. J., Jin, W., Greenwell-Wild, T., and Wahl, S. M. (2006). Induction of APOBEC3 family proteins, a defensive maneuver underlying 
interferon-induced anti-HIV-1 activity. J. Exp. Med. 203, 41-46. doi: 10.1084/jem.20051512

Pertel, T., Reinhard, C., and Luban, J. (2011). Vpx rescues HIV-1 transduction of dendritic cells from the antiviral state established by type 1 interferon. Retrovirology 8, 49. doi: 10.1186/1742-4690-8-49

Powell, R. D., Holland, P. J., Hollis, T., and Perrino, F. W. (2011). Aicardi-Goutieres syndrome gene and HIV-1 restriction factor SAMHD1 is a dGTP-regulated deoxynucleotide triphosphohydrolase. J. Biol. Chem. 286, 43596-43600. doi: 10.1074/jbc.C111.317628

Rajendra Kumar, P., Singhal, P. K., Subba Rao, M. R., and Mahalingam, S. (2005). Phosphorylation by MAPK regulates simian immunodeficiency virus Vpx protein nuclear import and virus infectivity. J. Biol. Chem. 280, 8553-8563. doi: 10.1074/jbc.M407863200

Rajendra Kumar, P., Singhal, P. K., Vinod, S. S., and Mahalingam, S. (2003). A non-canonical transferable signal mediates nuclear import of simian immunodeficiency virus Vpx protein. J. Mol. Biol. 331, 1141-1156. doi: 10.1016/S00222836(03)00853-2

Refsland, E. W., Stenglein, M. D., Shindo, K., Albin, J. S., Brown, W. L., and Harris, R. S. (2010). Quantitative profiling of the full APOBEC3 mRNA repertoire in lymphocytes and tissues: implications for HIV-1 restriction. Nucleic Acids Res. 38, 4274-4284. doi: 10.1093/nar/gkq174

Rehwinkel, J., Maelfait, J., Bridgeman, A., Rigby, R., Hayward, B., Liberatore, R. A., et al. (2013). SAMHD1-dependent retroviral control and escape in mice. $E M B O$ J. 32, 2454-2462. doi: 10.1038/emboj.2013.163

Reinhard, C., Bottinelli, D., Kim, B., and Luban, J. (2014). Vpx rescue of HIV-1 from the antiviral state in mature dendritic cells is independent of the intracellular deoxynucleotide concentration. Retrovirology 11, 12. doi: 10.1186/17424690-11-12

Rice, G. I., Bond, J., Asipu, A., Brunette, R. L., Manfield, I. W., Carr, I. M., et al. (2009). Mutations involved in Aicardi-Goutieres syndrome implicate SAMHD1 as regulator of the innate immune response. Nat. Genet. 41, 829-832. doi: $10.1038 /$ ng. 373

Roe, T., Reynolds, T. C., Yu, G., and Brown, P. O. (1993). Integration of murine leukemia virus DNA depends on mitosis. EMBO J. 12, 2099-2108.

Schwefel, D., Groom, H. C., Boucherit, V. C., Christodoulou, E., Walker, P. A., Stoye, J. P., et al. (2014). Structural basis of lentiviral subversion of a cellular protein degradation pathway. Nature 505, 234-238. doi: 10.1038/nature12815

Selig, L., Pages, J. C., Tanchou, V., Preveral, S., Berlioz-Torrent, C., Liu, L. X., et al. (1999). Interaction with the $\mathrm{p} 6$ domain of the gag precursor mediates incorporation into virions of $\mathrm{Vpr}$ and $\mathrm{Vpx}$ proteins from primate lentiviruses. J. Virol. $73,592-600$.

Sharova, N., Wu, Y., Zhu, X., Stranska, R., Kaushik, R., Sharkey, M., et al. (2008). Primate lentiviral Vpx commandeers DDB1 to counteract a macrophage restriction. PLoS Pathog. 4:e1000057. doi: 10.1371/journal.ppat.1000057

Sharp, P. M., Bailes, E., Stevenson, M., Emerman, M., and Hahn, B. H. (1996). Gene acquisition in HIV and SIV. Nature 383, 586-587. doi: 10.1038/383 $586 \mathrm{a} 0$

Sharp, P. M., and Hahn, B. H. (2011). Origins of HIV and the AIDS pandemic. Cold Spring Harb. Perspect. Med. 1:a006841. doi: 10.1101/cshperspect.a006841

Shibata, R., Miura, T., Hayami, M., Ogawa, K., Sakai, H., Kiyomasu, T., et al. (1990). Mutational analysis of the human immunodeficiency virus type 2 (HIV-2) genome in relation to HIV-1 and simian immunodeficiency virus SIV (AGM). J. Virol. 64, 742-747.

Shirazi, Y., and Pitha, P. M. (1992). Alpha interferon inhibits early stages of the human immunodeficiency virus type 1 replication cycle. J. Virol. 66, 1321-1328.

Shirazi, Y., and Pitha, P. M. (1993). Interferon alpha-mediated inhibition of human immunodeficiency virus type 1 provirus synthesis in T-cells. Virology 193, 303-312. doi: 10.1006/viro.1993.1126

Singhal, P. K., Kumar, P. R., Rao, M. R., Kyasani, M., and Mahalingam, S. (2006a). Simian immunodeficiency virus Vpx is imported into the nucleus via importin alpha-dependent and -independent pathways. J. Virol. 80, 526-536. doi: 10.1128/JVI.80.1.526-536.2006

Singhal, P. K., Rajendra Kumar, P., Subba Rao, M. R., and Mahalingam, S. (2006b). Nuclear export of simian immunodeficiency virus Vpx protein. J. Virol. 80, 12271-12282. doi: 10.1128/JVI.00563-06

Sonza, S., Maerz, A., Deacon, N., Meanger, J., Mills, J., and Crowe, S. (1996). Human immunodeficiency virus type 1 replication is blocked prior to reverse transcription and integration in freshly isolated peripheral blood monocytes. J. Virol. 70, 3863-3869.
Spragg, C. J., and Emerman, M. (2013). Antagonism of SAMHD1 is actively maintained in natural infections of simian immunodeficiency virus. Proc. Natl. Acad. Sci. U.S.A. 110, 21136-21141. doi: 10.1073/pnas.13168 39110

Srivastava, S., Swanson, S. K., Manel, N., Florens, L., Washburn, M. P., and Skowronski, J. (2008). Lentiviral Vpx accessory factor targets VprBP/DCAF1 substrate adaptor for cullin 4 E3 ubiquitin ligase to enable macrophage infection. PLoS Pathog. 4:e1000059. doi: 10.1371/journal.ppat.10 00059

Stetson, D. B., Ko, J. S., Heidmann, T., and Medzhitov, R. (2008). Trex1 prevents cell-intrinsic initiation of autoimmunity. Cell 134, 587-598. doi: 10.1016/j.cell.2008.06.032

St Gelais, C., De Silva, S., Amie, S. M., Coleman, C. M., Hoy, H., Hollenbaugh, J. A., et al. (2012). SAMHD1 restricts HIV-1 infection in dendritic cells (DCs) by dNTP depletion, but its expression in DCs and primary CD4+ T-lymphocytes cannot be upregulated by interferons. Retrovirology 9, 105. doi: 10.1186/17424690-9-105

Sze, A., Belgnaoui, S. M., Olagnier, D., Lin, R., Hiscott, J., and Van Grevenynghe, J. (2013). Host restriction factor SAMHD1 limits human T cell leukemia virus type 1 infection of monocytes via STING-mediated apoptosis. Cell Host Microbe 14, 422-434. doi: 10.1016/j.chom.2013.09.009

Triques, K., and Stevenson, M. (2004). Characterization of restrictions to human immunodeficiency virus type 1 infection of monocytes. J. Virol. 78, 5523-5527. doi: 10.1128/JVI.78.10.5523-5527.2004

Tristem, M., Marshall, C., Karpas, A., and Hill, F. (1992). Evolution of the primate lentiviruses: evidence from vpx and vpr. EMBO J. 11, 3405-3412.

Tristem, M., Marshall, C., Karpas, A., Petrik, J., and Hill, F. (1990). Origin of vpx in lentiviruses. Nature 347, 341-342. doi: 10.1038/347341b0

Trobaugh, D. W., Gardner, C. L., Sun, C., Haddow, A. D., Wang, E., Chapnik, E., et al. (2013). RNA viruses can hijack vertebrate microRNAs to suppress innate immunity. Nature 506, 245-248. doi: 10.1038/nature12869

Tungler, V., Staroske, W., Kind, B., Dobrick, M., Kretschmer, S., Schmidt, F., et al. (2013). Single-stranded nucleic acids promote SAMHD1 complex formation. J. Mol. Med. (Berl). 91, 759-770. doi: 10.1007/s00109-013-0995-3

Ueno, F., Shiota, H., Miyaura, M., Yoshida, A., Sakurai, A., Tatsuki, J., et al. (2003). Vpx and Vpr proteins of HIV-2 up-regulate the viral infectivity by a distinct mechanism in lymphocytic cells. Microbes Infect. 5, 387-395. doi: 10.1016/S1286-4579(03)00042-X

Vartanian, J. P., Guetard, D., Henry, M., and Wain-Hobson, S. (2008). Evidence for editing of human papillomavirus DNA by APOBEC3 in benign and precancerous lesions. Science 320, 230-233. doi: 10.1126/science.1153201

Wei, W., Guo, H., Gao, Q., Markham, R., and Yu, X. F. (2014). Variation of two primate lineage-specific residues in human SAMHD1 confers resistance to $\mathrm{N}$ terminus-targeted SIV Vpx proteins. J. Virol. 88, 583-591. doi: 10.1128/JVI.02866-13

Wei, W., Guo, H., Han, X., Liu, X., Zhou, X., Zhang, W., et al. (2012). A novel DCAF1-binding motif required for Vpx-mediated degradation of nuclear SAMHD1 and Vpr-induced G2 arrest. Cell. Microbiol. 14, 1745-1756. doi: 10.1111/j.1462-5822.2012.01835.x

Weiss, K. K., Chen, R., Skasko, M., Reynolds, H. M., Lee, K., Bambara, R. A., et al. (2004). A role for dNTP binding of human immunodeficiency virus type 1 reverse transcriptase in viral mutagenesis. Biochemistry 43, 4490-4500. doi: $10.1021 /$ bi035258r

Welbourn, S., Dutta, S. M., Semmes, O. J., and Strebel, K. (2013). Restriction of virus infection but not catalytic dNTPase activity are regulated by phosphorylation of SAMHD1. J. Virol. 87, 11516-11524. doi: 10.1128/JVI.01642-13

Wertheim, J. O., and Worobey, M. (2007). A challenge to the ancient origin of SIVagm based on African green monkey mitochondrial genomes. PLoS Pathog. 3:e95. doi: 10.1371/journal.ppat.0030095

White, T. E., Brandariz-Nunez, A., Valle-Casuso, J. C., Amie, S., Nguyen, L. A., Kim, B., et al. (2013). The retroviral restriction ability of SAMHD1, but not its deoxynucleotide triphosphohydrolase activity, is regulated by phosphorylation. Cell Host Microbe 13, 441-451. doi: 10.1016/j.chom.2013.03.005

Wu, X., Conway, J. A., Kim, J., and Kappes, J. C. (1994). Localization of the Vpx packaging signal within the $\mathrm{C}$ terminus of the human immunodeficiency virus type 2 Gag precursor protein. J. Virol. 68, 6161-6169.

Yan, J., Kaur, S., Delucia, M., Hao, C., Mehrens, J., Wang, C., et al. (2013). Tetramerization of SAMHD1 is required for biological activity and inhibition of HIV infection. J. Biol. Chem. 288, 10406-10417. doi: 10.1074/jbc.M112.443796 
Yu, H., Usmani, S. M., Borch, A., Kramer, J., Sturzel, C. M., Khalid, M., et al. (2013). The efficiency of Vpx-mediated SAMHD1 antagonism does not correlate with the potency of viral control in HIV-2-infected individuals. Retrovirology 10, 27. doi: 10.1186/1742-4690-10-27

Yu, X. F., Ito, S., Essex, M., and Lee, T. H. (1988). A naturally immunogenic virion-associated protein specific for HIV-2 and SIV. Nature 335, 262-265. doi: $10.1038 / 335262 \mathrm{a} 0$

Yu, X. F., Yu, Q. C., Essex, M., and Lee, T. H. (1991). The vpx gene of simian immunodeficiency virus facilitates efficient viral replication in fresh lymphocytes and macrophage. J. Virol. 65, 5088-5091.

Zhao, K., Du, J., Han, X., Goodier, J. L., Li, P., Zhou, X., et al. (2013). Modulation of LINE-1 and Alu/SVA retrotransposition by Aicardi-Goutieres syndrome-related SAMHD1. Cell Rep. 4, 1108-1115. doi: 10.1016/j.celrep.2013. 08.019

Zhao, L. J., Mukherjee, S., and Narayan, O. (1994). Biochemical mechanism of HIVI Vpr function. Specific interaction with a cellular protein. J. Biol. Chem. 269, $15577-15582$.
Conflict of Interest Statement: The authors declare that the research was conducted in the absence of any commercial or financial relationships that could be construed as a potential conflict of interest.

Received: 10 February 2014; paper pending published: 28 February 2014; accepted: 11 March 2014; published online: 08 April 2014.

Citation: Schaller T, Bauby H, Hué S, Malim MH and Goujon C (2014) New insights into an X-traordinary viral protein. Front. Microbiol. 5:126. doi: 10.3389/fmicb. 2014.00126

This article was submitted to Virology, a section of the journal Frontiers in Microbiology.

Copyright (c) 2014 Schaller, Bauby, Hué, Malim and Goujon. This is an open-access article distributed under the terms of the Creative Commons Attribution License (CC BY). The use, distribution or reproduction in other forums is permitted, provided the original author(s) or licensor are credited and that the original publication in this journal is cited, in accordance with accepted academic practice. No use, distribution or reproduction is permitted which does not comply with these terms. 